ARTICLE

\title{
Genomic and transcriptomic alterations associated with drug vulnerabilities and prognosis in adenocarcinoma at the gastroesophageal junction
}

\author{
Yuan Lin ${ }^{1,8}$, Yingying Luo (1) 2,8, Yanxia Sun (1) 2,8, Wenjia Guo (1) 2,3,8, Xuan Zhao 2,8, Yiyi Xi ${ }^{2}$, Yuling Ma(1) ${ }^{2}$, \\ Mingming Shao ${ }^{2}$, Wen Tan ${ }^{2}$, Ge Gao (1) ${ }^{1,4 凶}$, Chen Wu (i) ${ }^{2,5,6 凶}$ \& Dongxin $\operatorname{Lin}^{2,5,7}$
}

Adenocarcinoma at the gastroesophageal junction (ACGEJ) has dismal clinical outcomes, and there are currently few specific effective therapies because of limited knowledge on its genomic and transcriptomic alterations. The present study investigates genomic and transcriptomic changes in ACGEJ from Chinese patients and analyzes their drug vulnerabilities and associations with the survival time. Here we show that the major genomic changes of Chinese ACGEJ patients are chromosome instability promoted tumorigenic focal copynumber variations and COSMIC Signature 17-featured single nucleotide variations. We provide a comprehensive profile of genetic changes that are potentially vulnerable to existing therapeutic agents and identify Signature 17-correlated IFN- $\alpha$ response pathway as a prognostic marker that might have practical value for clinical prognosis of ACGEJ. These findings further our understanding on the molecular biology of ACGEJ and may help develop more effective therapeutic strategies.

\footnotetext{
${ }^{1}$ Beijing Advanced Innovation Center for Genomics (ICG), Biomedical Pioneering Innovation Center (BIOPIC), Peking University, Beijing, China. ${ }^{2}$ Department of Etiology and Carcinogenesis, National Cancer Center/National Clinical Research Center/Cancer Hospital, Chinese Academy of Medical Sciences and Peking Union Medical College, Beijing, China. ${ }^{3}$ Cancer Institute, Affiliated Cancer Hospital of Xinjiang Medical University, Urumqi, China. ${ }^{4}$ State Key Laboratory of Protein and Plant Gene Research, School of Life Sciences, Center for Bioinformatics, Peking University, Beijing, China. ${ }^{5}$ Collaborative Innovation Center for Cancer Personalized Medicine, Nanjing Medical University, Nanjing, China. ${ }^{6}$ CAMS Key Laboratory of Genetics and Genomic Biology, Chinese Academy of Medical Sciences and Peking Union Medical College, Beijing, China. ${ }^{7}$ Sun Yat-sen University Cancer Center, State Key Laboratory of Oncology in South China, Guangzhou, China. ${ }^{8}$ These authors contributed equally: Yuan Lin, Yingying Luo, Yanxia Sun, Wenjia Guo, Xuan Zhao.

凶email: gaog@mail.cbi.pku.edu.cn; chenwu@cicams.ac.cn
} 
$\mathrm{n}$ the past few decades, the incidences of adenocarcinoma at the gastroesophageal junction (ACGEJ) are rapidly increasing worldwide ${ }^{1,2}$. While it can be categorized as esophageal or gastric adenocarcinoma ${ }^{3}$, ACGEJ has been treated as the latter in Chinese hospitals because esophageal cancer in China is mostly squamous-cell carcinomas ${ }^{4}$. Surgical resection is the standard treatment for early-stage ACGEJ and for locally advanced or unresectable tumors, pre- and post-operative chemotherapies are often used ${ }^{5}$. Targeted therapies are only for patients with latestage metastatic HER2-positive tumors ${ }^{6}$, although Nivolumab, an immuno-oncology agent targeting PD-L1, has showed encouraging efficacy in patients with unresectable advanced or recurrent HER2-negative ACGEJ ${ }^{7}$. The 5-year survival rates of this cancer are $20-25 \%$, lower than that of esophageal or gastric cancers. Thus, it is urgent to apprehend molecular characteristics that can serve as potential drug targets and (or) prognostic indicators.

In a previous study conducted in Caucasian patients by the Cancer Genome Atlas (TCGA) project $^{8}, 65 \%$ of ACGEJ were categorized as the chromosomal instability (CIN) subtype of gastric cancer, characterized by preponderating focal copynumber variations (CNVs) in the tumor genome. Somatic single nucleotide variations (SNVs) and small insertions and deletions (indels) significantly recur in TP53 and genome-wide single base substitutions (SBSs) typically form a pattern known as Signature 17 in the Catalogue of Somatic Mutations in Cancer (COSMIC) $)^{9,10}$. Signature 17 may be the footprint of an early mutational mechanism initiating esophageal and gastric adenocarcinoma ${ }^{11}$, and its characteristic SBS, $5^{\prime}-\mathrm{C}[\mathrm{T}>\mathrm{G}] \mathrm{T}-3^{\prime}$, has been associated with poor survival of esophageal adenocarcinoma ${ }^{12}$.

Due to different genetic makeup and environments including lifestyles, whether ACGEJ genomes in Chinese patients share the above characteristics is unclear. Previous gastric cancer studies on East Asian patients have each collected about 30 ACGEJ samples ${ }^{13,14}$, probably too few to draw rigorous conclusions. Moreover, previous studies did not provide enough information on transcriptomic changes that could further their significant findings on the genomic changes of ACGEJ. In the present study, we have assembled a relatively large set of Chinese ACGEJ patients and have sequenced genomes and transcriptomes of matched tumor and adjacent normal tissue samples. By jointly analyzing these data and comparing with the findings in Caucasian patients, we show that ACGEJ in Chinese patients are also dominated by CIN-associated focal CNVs and that Signature 17 activities correlate with multiple essential genomic and transcriptomic changes of ACGEJ. Furthermore, we deliver a comprehensive profile of genetic alterations potentially vulnerable to existing treatments, and identify genomic and transcriptomic prognostic markers of potential clinical values. These findings may improve current knowledge about ACGEJ and contribute to its precision diagnosis and treatment.

\section{Results}

$\mathrm{CIN}$-associated focal $\mathrm{CNV}$ is the major feature of ACGEJ genomic alterations. We performed whole-genome sequencing (WGS) on ACGEJ tumor samples containing $\geq 60 \%$ of cancer cells (Supplementary Fig. 1a) and matched blood samples from 124 Chinese patients (mean coverage $61 \mathrm{x}$ and $31 \mathrm{x}$, respectively) and identified 2,558,269 SNVs and 1,258,899 indels. The tumor mutation burden (TMB) ranged from 0 to 13.1 (median 1.8) per megabase $(\mathrm{Mb})$. The most significantly mutated gene was TP53 (FDR $q \leq 0.05)$ with coding mutations found in $71.0 \%(88 / 124)$ samples. To increase the detection power, we combined the reported coding-region mutations data of 151 ACGEJ samples $8,15,16$ with our data and found that TP53 was the only gene recurrently mutated in $\geq 10 \%$ of samples (Supplementary
Table 1). CNVs overlapped with a median of $7.8 \%$ of the tumor genome and $8.4 \%$ of the coding region, respectively (Supplementary Fig. 2a) and the jagged layout of genome-wide CNV distributions (Fig. 1a) indicates widespread focal CNVs. Proteincoding genes subject to CNVs were 5.7 times (median) more than those altered by non-silent SNVs/indels (Fig. 1b). These results suggest that focal CNVs are the major genomic alterations of ACGEJ in Chinese patients.

Since $65 \%$ of TCGA ACGEJ samples were deemed CIN and $\mathrm{CIN}$ is a common source of CNVs in cancer genomes associated with metastasis, therapeutic resistance, and immune evasion ${ }^{17,18}$, we investigated the prevalence of CIN in our samples. We found a published CIN gene signature (CIN70) ${ }^{19}$ significantly overexpressed in tumors compared with adjacent normal tissues $(P=0.001$; Fig. 1c) and whole-genome doubling (WGD), a known precursor to $\mathrm{CIN}^{20}$, in $59.7 \%(74 / 124)$ of our ACGEJ genomes. We also found other genomic abnormalities suggestive of CIN including chromothripsis $(n=77,54$ with WGD), kataegis $(n=74)$, and complex structural variations (SVs) such as translocations (median 141.5 per ACGEJ genome) and inversions (median 257.5 per tumor genome) (Supplementary Data 1,2). The expression levels of CIN70 were significantly higher in WGD than in non-WGD tumor genomes (median 0.50 versus $0.28, P=2.15 \mathrm{e}-5$ ) and significantly correlated with the number of chromosomal arm and gene level CNVs (Spearman's $\rho=0.30$ and $0.27, P=6.60 \mathrm{e}-4$ and 0.003 , respectively) (Fig. 1d). ACGEJ genomes with WGD also had more gene level CNVs than those without WGD (median 609.5 versus $312, P=8.01 \mathrm{e}-4$ ). The median ploidy of WGD genomes was 3.1 and they had a significantly larger proportion of autosomal genome losing heterozygosity than non-WGD genomes (39\% versus $25 \%, P=$ $2.43 \mathrm{e}-6)$, indicating frequent single-copy losses after WGD. Together, these results suggest the abundant CNVs observed in our ACGEJ genomes were associated with CIN.

The genomic regions of significantly recurrent CNVs (FDR $q \leq 0.1$, in $\geq 10 \%$ samples) harbored 25 oncogenes or tumor suppressor genes (TSGs) knowingly affected by $\mathrm{CNVs}^{21,22}$ (Fig. 1e) including CCNE1, RICTOR, VEGFA, ERBB2, FGFR2, $B C L 2 L 1, C D K 6, E R B B 3, M E T, C D H 1, A R I D 1 A, A P C$, and $C D K N 2 B$ that had correlated copy-number and expression changes (Spearman's $\rho \geq 0.3$, FDR $q<0.002$ ) (Supplementary Fig. 2b). CNVs of these 13 genes are potential ACGEJ drivers, of which CCNE1 and ERBB2 amplifications occurred most frequently ( $n=35$ and $24 ; 28.2 \%$ and $19.4 \%$, respectively) and significantly co-existed $(n=13,10.5 \% ; P=0.004)$. We found genomic and transcriptomic evidence suggesting an association between the dysfunction of CCNE1 and CIN. ACGEJ with CCNE1 copy number gains $(n=67,54.0 \%)$ had more CNVs at both chromosomal arm level (median 21 versus $14, P=3.20 \mathrm{e}-5$ ) and gene level (median 618 versus $251, P=8.88 \mathrm{e}-5$ ), and were more likely to undergo WGD $(P=4.28 \mathrm{e}-4)$ than ACGEJ without CCNE1 gains $(n=57,46.0 \%)$ (Fig. 1f); these associations remained significant after adjusting for TP53 mutation status $(P<0.02)$. The expression levels of $C C N E 1$ were highly correlated with CIN70 activities (Spearman's $\rho=0.53, P=3.93 \mathrm{e}-10)$ and significantly elevated in WGD tumor samples (fold change $=$ $1.21, P=0.011$ ) (Fig. 1g). Consistently, CCNE1 amplification has been associated with WGD in TCGA pan-cancer analyses ${ }^{23,24}$ and CCNE1 overexpression has been shown to induce CIN phenotypes in various cancer cells ${ }^{25,26}$.

COSMIC Signature 17 is the characteristic ACGEJ mutational signature. We next examined the mutation spectra of our ACGEJ genomes to characterize the mutational signature and found $5^{\prime}-\mathrm{C}$ $[\mathrm{T}>\mathrm{G}] \mathrm{T}-3^{\prime}$ was the most common somatic SBS across the 
a

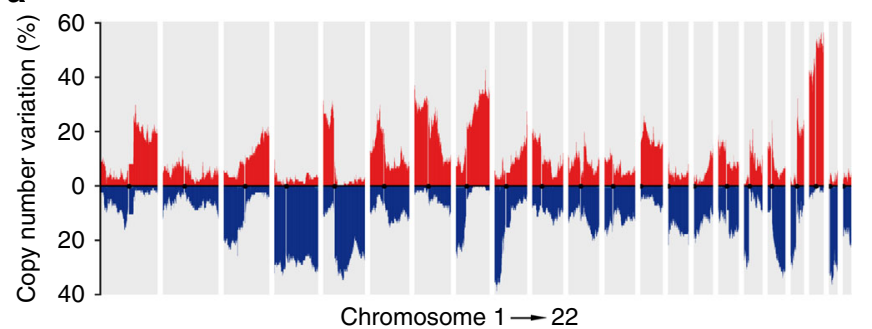

b

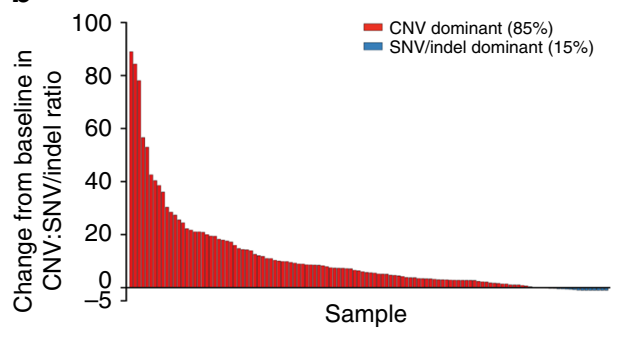

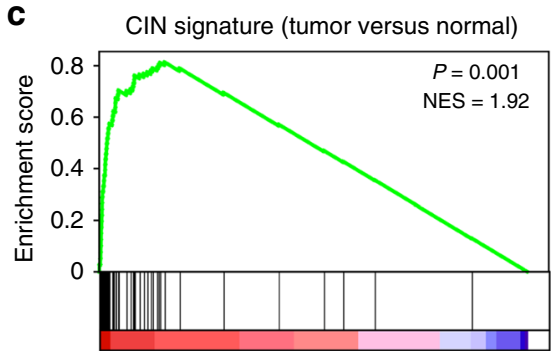
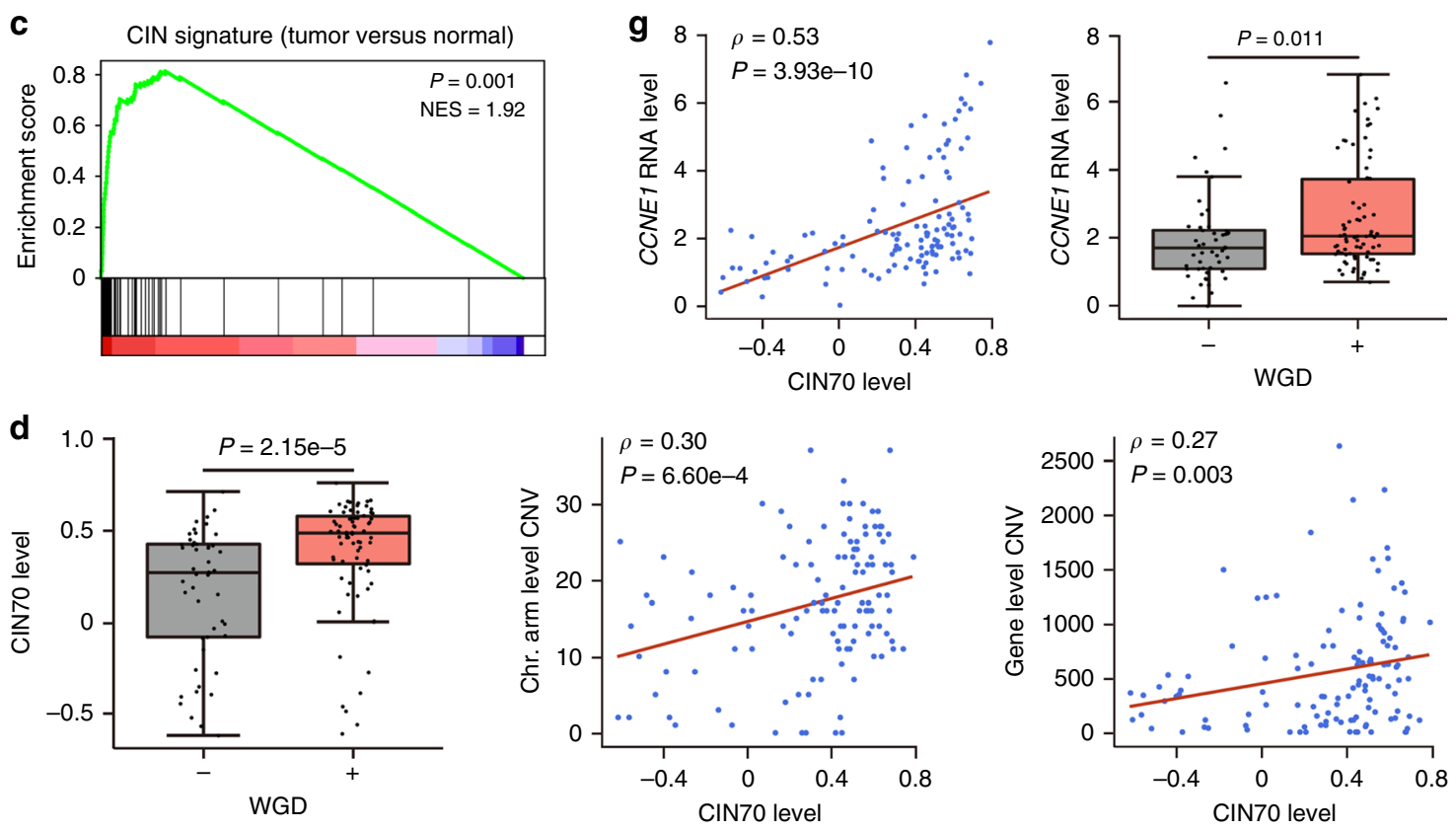

e
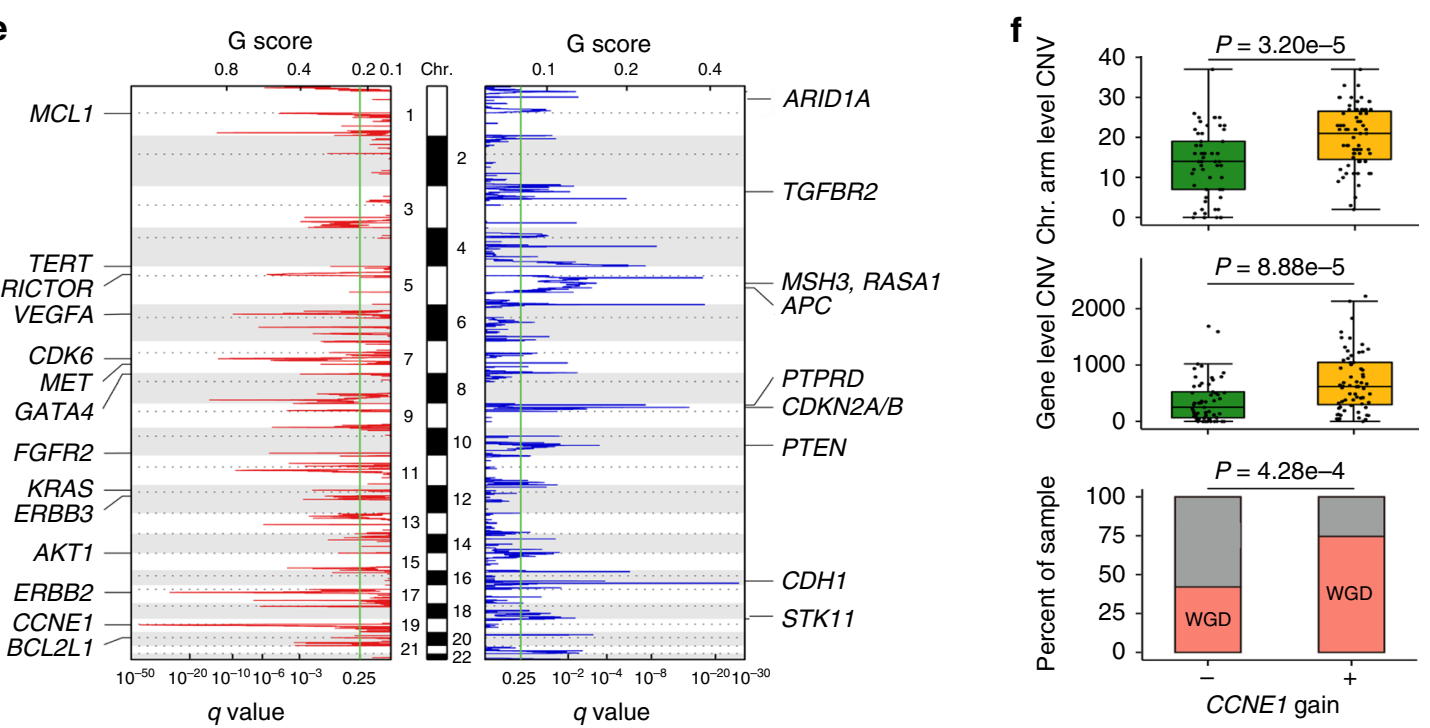

genomes (Fig. 2a). When fitted to reported COSMIC mutational signatures, this SBS becomes a major component of Signature 17. We also found recurrent activities of COSMIC Signatures 1, 3, 5, and 8 (Fig. 2b). In the ACGEJ genomes of our patients, $52.3 \%$ $(257,673 / 493,106)$ of Signature 17 attributed SNVs were located at intergenic regions. We searched for potential cancer-driving regulatory elements in these regions and found Signature 17 SNVs in $75.3 \%(314 / 417)$ of significantly mutated CTCF binding sites $^{27}$ (Supplementary Data 3). Because oncogenic SNVs at
CTCF binding sites have been linked to $\mathrm{CIN}^{27-29}$, we then investigated correlations between Signature 17 and CINrelated genomic alterations. Signature 17 activities were higher in ACGEJ genomes with TP53 coding mutations or WGD or chromothripsis than in genomes without these features $(P=$ $0.002,0.006$, and 0.009 , respectively) and were positively correlated with TMB and the number of chromosomal arm level CNVs (Spearman's $\rho=0.26$ and $0.32, P=0.004$ and $2.57 \mathrm{e}-4$, respectively; Fig. 2c, d). 
Fig. 1 Featured ACGEJ genomic and transcriptomic changes. a Frequencies of CNVs detected in 124 ACGEJ samples, with gains in red and losses in blue. Bars represent non-overlapped 1-million-base-pair windows along the genome. b Bar plot comparing the number of genes altered by CNVs and SNVs/ indels in each of 120 ACGEJ genomes with both types of alterations. The $y$-axis indicates the ratio of CNVs to SNVs/indels minus 1 . Only deletions, amplifications, or non-silent coding mutations are counted. Most samples (102/120, 85\%) show a preponderance of CNVs over SNVs/indels. c GSEA analysis comparing CIN70 activities in ACGEJ samples and adjacent normal tissue samples. The plot shows overexpressed CIN70 in ACGEJ samples, the normalized enrichment score (NES) and the $P$ value. $\mathbf{d}$ Associations between CIN70 activities and the WGD status ( + and - indicating 74 and 50 tumor genomes with and without WGD, respectively; two-sided Wilcoxon rank-sum test), the number of chromosomal (Chr.) arm or gene level CNVs (Spearman's correlation tests) in ACGEJ samples. e GISTIC2.0 identified recurrent focal CNVs in 124 ACGEJ genomes, with 25 potential CNV drivers annotated on the plot. $\mathbf{f}$ Box and bar plots comparing chromosomal (Chr.) arm level CNVs, gene level CNVs, and the frequencies of WGD in tumor genomes with and without CCNE1 copy number gains ( $n=67$ and 57, respectively; two-sided Wilcoxon rank-sum tests). $\mathbf{g}$ Associations between CCNE1 expression levels and CIN70 activities (Spearman's correlation test) or WGD status (two-sided Wilcoxon rank-sum test) in ACGEJ samples. Box plots in (d, $\mathbf{f}, \mathbf{g}$ ) show the median (central line), the $25-75 \%$ interquartile range (IQR) (box limits), the \pm 1.5 times IQR (Tukey whiskers), and all data points, among which the lowest and the highest points indicate minimal and maximal values, respectively.

We further investigated Signature 17-correlated transcriptomic changes. Since the underlying mutational process represented by Signature 17 has not been well established, we sought for genes whose expressions are correlated with Signature 17 activities and assessed the enrichment of these genes in cancer hallmark pathways and gene signatures (see Methods). Among pathways and gene signatures differentially expressed between our tumor and adjacent normal samples (FDR $q \leq 1 \mathrm{e}-4$ ), the CIN70 gene signature was enriched with genes positively correlated with Signature 17 activities (e.g., TPX2, NEK2, and KIF4A). Additionally, the mitotic cell-cycle, mitotic spindle, E2F targets, and G2M checkpoint pathways were also enriched with genes positively correlated with Signature 17 (e.g., MYBL2, KIF15, and CCNE1), suggesting elevated cell proliferation of tumors with high Signature 17 activities. Immunity-related pathways including allograft rejection, interferon (IFN)- $\alpha$ or IFN- $\gamma$ response, and the IL6-JAK-STAT3 signaling were down-regulated in tumor samples and they were enriched with Signature 17 negatively correlated genes (e.g., CXCR3, A2M, and FGL2), suggesting suppressed immune response in Signature 17-high tumors. We also found the oxidative phosphorylation and glycolysis pathways were, respectively, enriched with Signature 17 negatively and positively correlated genes (e.g., CLDN3, NDUFA4L2, and UQCRC2), suggesting metabolic remodeling in Signature 17-high tumors (Fig. 2e).

Similarly, assessing the enrichment of Signature 17-correlated genes in a set of marker genes for CD $8+\mathrm{T}, \gamma \delta \mathrm{T}$, and natural killer (NK) cells ${ }^{30}$ revealed decreased cytotoxic cell activities in Signature 17-high tumors (Fig. 2e). We estimated the fractions of ten immune cell types to total cells in ACGEJ and adjacent normal samples using our bulk RNA sequencing data and found CD8 $+\mathrm{T}$ and NK cells present $(>0 \%)$ in $97.6 \%(120 / 123)$ and $98.4 \%(121 / 123)$ tumor samples, respectively, but the most prevalent immune cell types in tumors were immune suppressive regulatory $\mathrm{T}$ cells (Treg, median 5.49\%). Signature 17 activities were negatively correlated with the faction of $\mathrm{CD} 8+\mathrm{T}$ cells (partial Spearman's $\rho=-0.16, P=9.95 \mathrm{e}-6)$. Compared with normal samples, tumor samples had significantly increased fractions of CD8 $+\mathrm{T}(1.46-1.80 \%, P=0.006)$ and NK cells $(0.80-1.37 \%, P=3.65 \mathrm{e}-13)$, and decreased fractions of neutrophils (4.28-3.07\%, $P=5.44 \mathrm{e}-6)$ (Fig. 2f). Although the proportions of M1 and M2 macrophages both increased (1.52-4.76\% and $1.60-2.79 \%, P=1.96 \mathrm{e}-20$ and $4.04 \mathrm{e}-5$, respectively), the $\mathrm{M} 1 /$ M2 ratio was significantly higher in tumor than in normal samples (1.70 versus $0.71, P=1.15 \mathrm{e}-13)$. However, immune checkpoint genes including IDO1, HAVCR2, PDCD1LG2, CD274, CTLA4, and TIGIT were significantly up-regulated in tumor samples (fold change $>1.30$, FDR $q \leq 1.0 \mathrm{e}-5$ ). These results collectively point to an immunosuppressive microenvironment of ACGEJ, which is even worse in Signature 17-high tumors.
Comparative analysis on major genomic alterations of our patients and TCGA patients. We compared clinically relevant characteristics of ACGEJ samples collected by this study $(n=$ $124)$ and by TCGA $(n=105)$. Due to the controlled access of TCGA Level 1/2 data, we downloaded Level 3 data and applied the same downstream analyses. The patients enrolled in this study were slightly younger than TCGA patients (median 65 and 66 years, respectively, $P=0.050)$ and exhibited more advanced (stage III or IV) ACGEJ (72 and 41 patients, respectively, $P=$ 0.021). While two cohorts shared similar sex distributions, the TCGA cohort was more ethnically diverse. Eighty-eight ancestryknown TCGA patients contain 78 Caucasians, nine Asians, and one African American. Five TCGA ACGEJ samples were likely microsatellite instable, while no such samples were identified in our cohort. Disregarding these samples, the TMB of the rest of TCGA ACGEJ $(n=100)$ was still higher than that of ours (median 2.68 and 1.84, respectively). The recurrence rates of functional mutations (annotated as oncogenic or likely oncogenic by OncoKB $^{21}$ ) in TP53, PTEN, ARID1A, CDKN2A, and KRAS appeared to be significantly higher in TCGA patient cohort than in our patient cohort. We did not find CDKN2A and KRAS mutations in our samples but detected $4.0 \%$ of LIPF mutations absent in TCGA samples (Supplementary Table 2).

Our samples were not significantly different from TCGA samples in the number of chromosomal arm level CNVs (median 17 for both) or gene level CNVs (median 423.5 and 370, respectively). High correlations of the cohort-level recurrences of arm level CNVs (Fig. 3a) indicated similar arm level CNV patterns across two cohorts. The genome-wide distributions of focal CNV regions were also similar in terms of recurrences and amplitudes (Fig. 3b). We assessed the overlaps between significantly recurrent focal CNVs (FDR $q<0.25$ ) across two cohorts and found $46.4 \%$ of focal amplifications and $50.8 \%$ of focal deletions identified from our samples had $\geq 50 \%$ overlap with those identified from TCGA samples. We then searched for $\mathrm{CNV}$ driver genes in highly recurrent focal $\mathrm{CNV}$ regions identified in either cohort; any two regions identified from different cohorts and reciprocally overlapping at $\geq 50 \%$ of both their sizes were merged into one big region and considered shared by both cohorts. In these regions, we found 28 genes with highly correlated copy-number and expression changes (Spearman's $\rho \geq$ 0.3 , FDR $q<0.05$ ) in respective cohorts (Fig. 3c). Homologous deletions or amplifications of these genes, which might drive ACGEJ, occurred mostly at comparable frequencies between two cohorts (Fig. 3d), except for more frequent CCNE1 and BCL2L1 amplifications in our samples $(P=0.016$ and $P=0.007$, respectively).

We then compared the impacts of potentially functional alterations (i.e., oncogene amplification, TSG homologous deletion, and functional mutations in both) at the pathway level. In 

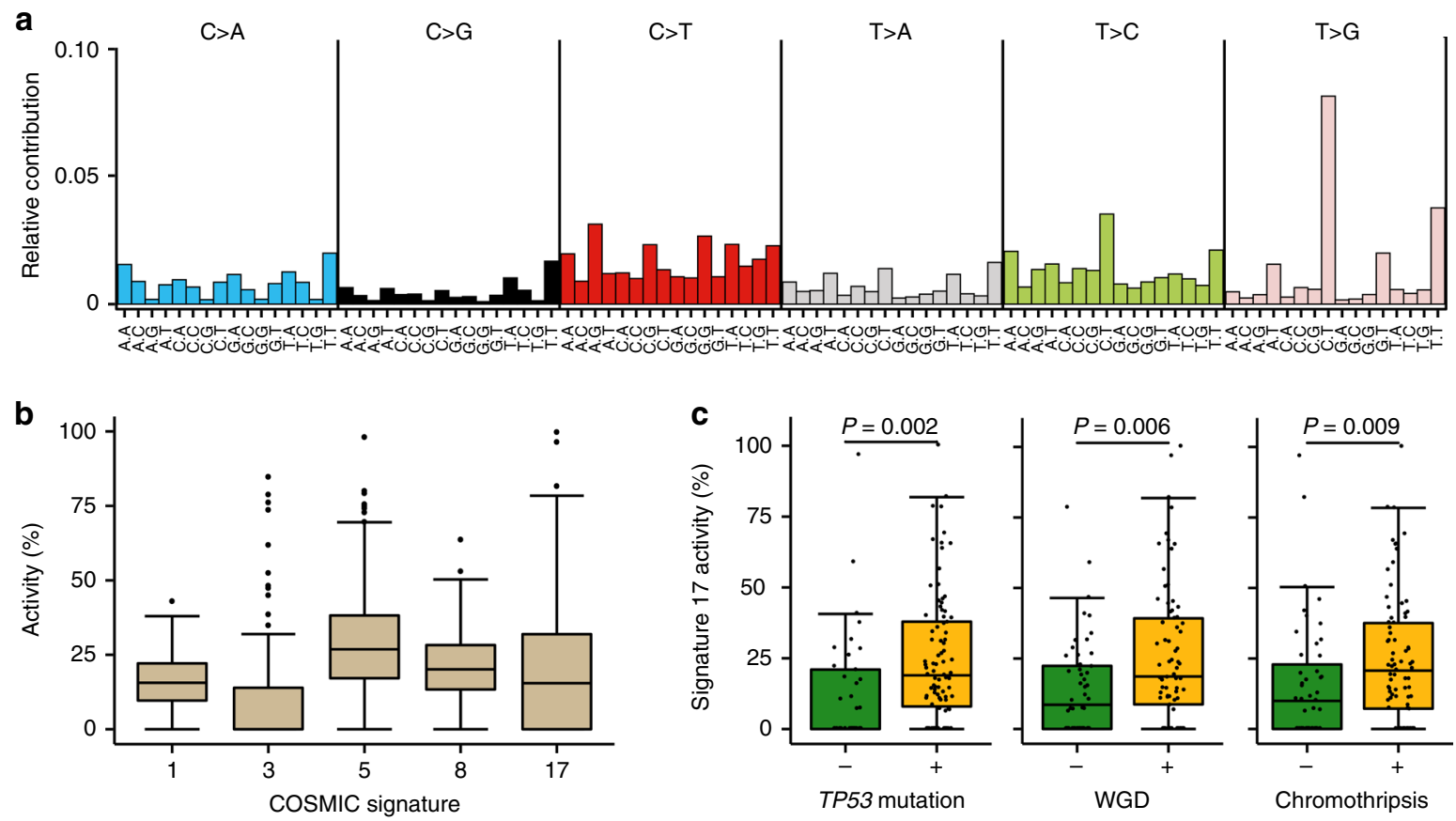

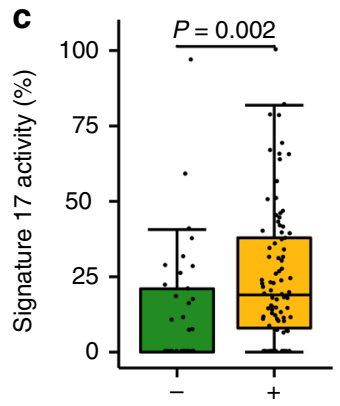

TP53 mutation

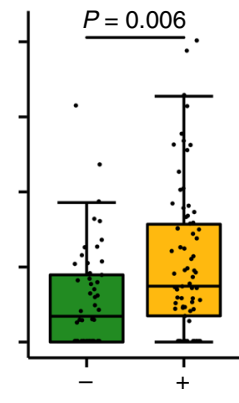

WGD

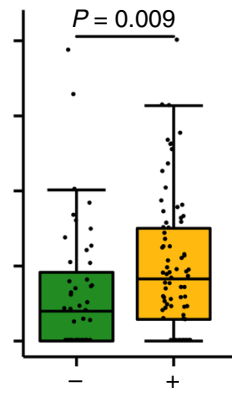

Chromothripsis
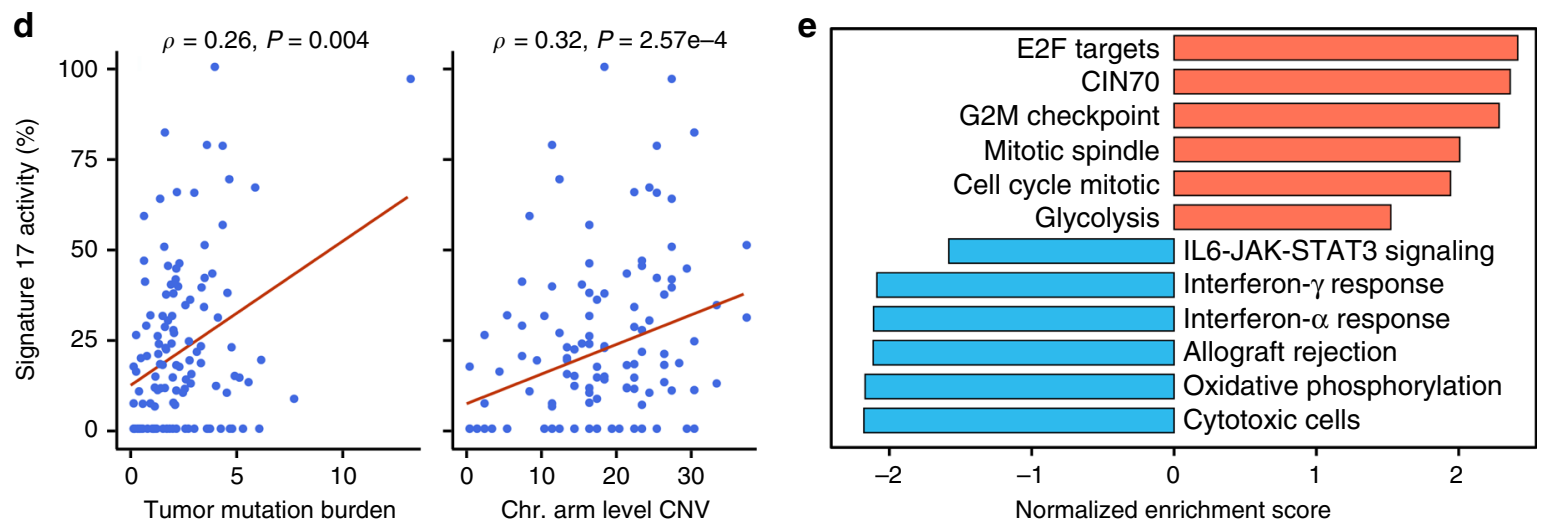

$\mathbf{f}$

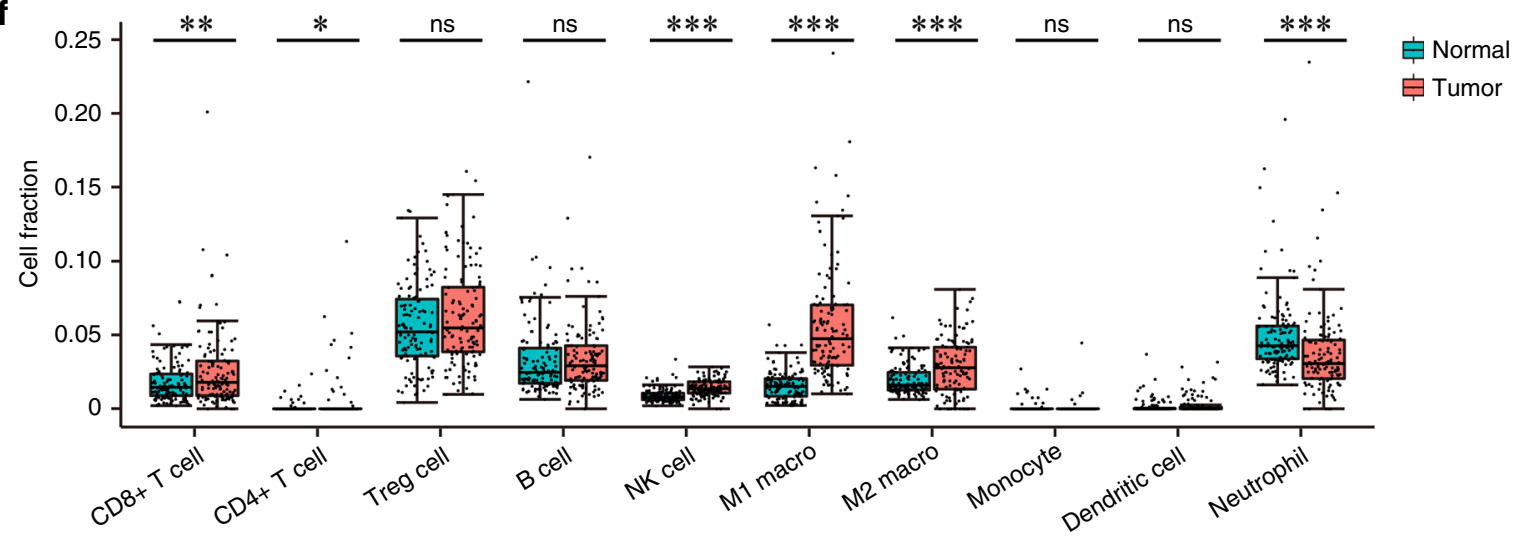

Fig. 2 Genomic and transcriptomic features associated with COSMIC Signature 17. a Mutational spectra of our ACGEJ samples. Different colors represent six SBS categories. On the $x$-axis, 16 possible trinucleotide contexts are repeated for each SBS category. b Distributions of COSMIC Signatures 1 , $3,5,8$, and 17 activities in 124 ACGEJ genomes. c Box plots comparing Signature 17 activities in ACGEJ samples with and without TP53 mutations ( $n=88$ and 36, respectively), WGD ( $n=74$ and 50, respectively), or chromothripsis ( $n=77$ and 47 , respectively); $P$ values derived from two-sided Wilcoxon rank-sum tests. $\mathbf{d}$ Spearman's correlations between Signature 17 activities and TMB or chromosomal (Chr.) arm level CNVs in ACGEJ. e Pathways or gene signatures enriched with genes positively (red bars) or negatively (blue bars) correlated with Signature 17 activities. $\mathbf{f}$ Box plots comparing the fractions of ten immune cell types in total cells between 124 pairs of ACGEJ and matched adjacent normal tissue samples. ${ }^{\star} P<0.05$; ${ }^{\star \star} P<0.01$; ${ }^{\star \star \star} P<0.001$; ns, not significant of two-sided Wilcoxon rank-sum test; macro., macrophage. Box plots show the median (central line), the 25-75\% interquartile range (IQR) (box limits), the \pm 1.5 times IQR (Tukey whiskers), and outliers (b) or all data points $(\mathbf{c}, \mathbf{f})$; the lowest and the highest data points indicate minimal and maximal values, respectively. 

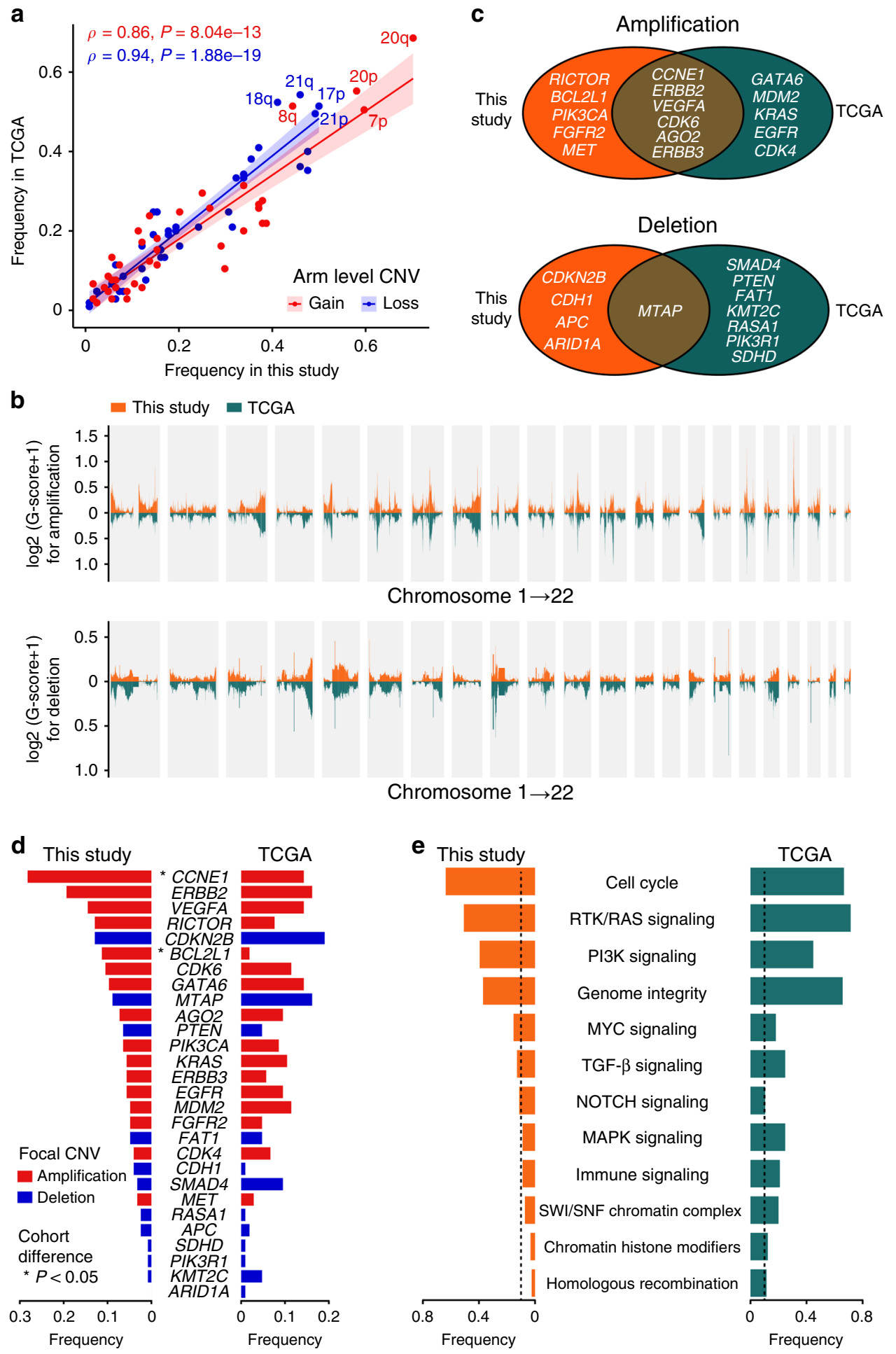

Fig. 3 Comparison on genomic alterations between our patients and TCGA patients. a Correlations of arm level CNV frequencies between our tumor samples and TCGA samples. Red and blue represent arm level gain and loss, respectively. b Bar plots comparing log2-tranformed GISTIC G-scores, a measure for both frequency and amplitude, of focal copy-number gain (upper panel) and loss (lower panel) regions in our tumor samples and TCGA samples. c Shared and unique putative CNV driver genes targeted by recurrent focal copy-number gains (upper panel) and losses (lower panel) in our samples and TCGA samples. d Bar plots comparing amplification (red) and deletion (blue) frequencies of putative CNV driver genes (c) across two cohorts. Genes with significantly different alteration frequencies in two cohorts $(P<0.05)$ were asterisked. e Bar plots comparing the frequencies of featured pathway perturbations across two cohorts. Dashed lines indicate $10 \%$ alteration frequency.

both cohorts, we found recurrent perturbations ( $>10 \%$ samples) to the cell-cycle, receptor tyrosine kinases (RTK)/RAS, PI3K, MYC, TGF- $\beta$, and NOTCH signaling pathways, and to genome integrity related genes (e.g., TP53, MDM2, and ERCC2), with different rates (Fig. 3e). In addition, MAPK signaling (e.g., KRAS, $M A P 2 K 1$ ), immune signaling (e.g., $H L A-A / B)$, the SWI/SNF chromatin remodeling complex (e.g., ARID1A, SMARCA4), chromatin histone modifiers (e.g., EP300, KMT2C), and the 
homologous recombination pathway (e.g., BRCA1, TP53BP1) were recurrently perturbed in the TCGA cohort but not in ours. Despite different genomic perturbation rates, the activities of these pathways or gene sets in our tumor samples and TCGA samples, as quantified by Gene Set Variation Analysis (GSVA) ${ }^{31}$ scores using normalized and batch corrected gene expression data, were similar with no statistically significant difference. So were the estimated fractions of different immune cell types. Together, these results suggest that our patient set and TCGA patient set had similar genomic alterations with some differences.

Genomic alterations indicate potential vulnerability to existing therapeutic agents. The lack of specific and effective drugs for ACGEJ treatments is largely due to our limited knowledge on the genomic characteristics of this disease. Thus, we screened our ACGEJ samples for genomic alterations predicting vulnerabilities to therapeutic agents approved by FDA or NCCN, currently in clinical trials, or supported by evidence from pre-clinical assays or case reports ${ }^{32}$. This analysis revealed 488 alterations in 67 genes with potential responses to 66 classes of 93 drugs (Supplementary Data 4). We predicted the genetic vulnerabilities of 105 TCGA ACGEJ samples with the same approach (Supplementary Data 5). For cytotoxic chemotherapeutic agents, we predicted that 96 of our patients would respond to six classes of drugs, including Anthracyclines, Gemcitabine, Mytomycin C, Temozolomide, Platinum agents, and Vinblastine (Fig. 4a). Anthracyclines, Gemcitabine, and Mytomycin C might have substantially higher response rates in our patients $(71.0-73.4 \%, 88-91$ of 124$)$ than in TCGA ACGEJ patients (49.5-57.1\%, 52-60 of 105) (Fig. 4a). The vulnerabilities of our patients to the three drugs were predicted mainly based on coding mutations in TP53. Anthracyclines has also been tested in a clinical trial as an inhibitor of TOP $2 A^{33}$, which was amplified in $11.3 \%(14 / 124)$ of our patients. We found significantly more frequent CIN-related genomic alterations (i.e., WGD, chromothripsis, and kataegis) in patients predictively responsive to any of the three drugs $(n=91)$ than in other patients ( $P=2.00 \mathrm{e}-5,0.035$, and $2.00 \mathrm{e}-5$, respectively) (Fig. $4 \mathrm{~b})$, consistent with a previous report that CIN-type gastric cancer is sensitive to adjuvant chemotherapy ${ }^{34}$.

For targeted therapeutic agents (Fig. 4c, d), 19.4\% (24/124) of our patients had ERBB2 amplification and therefore could benefit from FDA approved ERBB2 inhibitors, including Trastuzumab, Pertuzumab, Neratinib, Lapatinib, and Ado-Trastuzumab Emtansine. Of the 100 patients without ERBB2 amplifications, 89 had other genomic alterations likely druggable by 59 classes of agents. Specifically, we predicted that WEE1, CDK4/6, and PARP inhibitors would be effective for 70,42 , and 27 of our patients, respectively, and together they could cover $93.3 \%(83 / 89)$ of our patients without ERBB2 amplification. Among potentially actionable gene alterations found in all 124 patients (Fig. 4c), the TP53 mutations in 88 patients were the major contributors to the predicted efficacy of WEE1 inhibitors. While only 19 patients had CDK4/CDK6 alterations, other alterations such as CDKN2A/ CDKN2B deletions or CCND1 amplifications in another 29 patients might also render them vulnerable to CDK4/6 inhibitors. Using the RNA sequencing data of paired ACGEJ and adjacent normal tissue samples of each patient, we found that $85.2 \%$ (404/ 474) of the predictively targetable gene alterations had corresponding expression changes (Supplementary Data 6). Recurrent inconsistency ( $>10$ samples) was only found for FGF3 and FGF4 amplifications (in 13 and 14 samples, respectively), potentially affecting the predicted response rates of two FGFR inhibitors Lucitanib and Dovitinib.

We compared the classes of targeted therapeutic agents with $\geq 20 \%$ predicted response rates in our 89 patients and 85 TCGA
ACGEJ patients that had no ERBB2 amplifications (Fig. 4d) and found that of the eight guideline or clinical-trial drug classes (i.e., WEE1, CDK4/6, PARP, FGFR, PI3K, AKT, AURKA-VEGF, and MTOR inhibitors), WEE1 and FGFR inhibitors had higher whereas others had lower response rates in our patients than in TCGA patients. Since CCNE1 was recurrently amplified, overexpressed, and often co-amplified with ERBB2 in our tumor samples, we took a special interest in druggable CDK2 whose activity is regulated by Cyclin E1 produced by CCNE1. The predicted response rate to existing CDK2 inhibitors was 29.8\% (37/124) in all our patients and $24.7 \%(22 / 89)$ in our patients without ERBB2 amplifications, compared with $16.2 \%(17 / 105)$ and $15.3 \%(13 / 85)$ in TCGA ACGEJ patients, respectively.

Unfortunately, 11 of our patients had no potentially druggable gene alterations. The tumor genomes of these patients had significantly lower TMB (median 1.87 versus 0.06 per $\mathrm{Mb}, P=$ $1.81 \mathrm{e}-4$ ), fewer CNVs at the chromosomal arm level (median 1 versus $17, P=7.84 \mathrm{e}-6)$ and the gene level (median 0 versus 490 , $P=6.10 \mathrm{e}-6)$ and less frequent WGD $(2 / 11$ versus $72 / 113, P=$ 0.007 ) than the tumor genomes of other patients (Fig. 4e). Based on the hematoxylin and eosin (H\&E)-stained histopathological images of these 11 patients' tumor samples (Supplementary Fig. 1b), we estimated that the range of their tumor cell contents were $60-95 \%$, which eliminated the possibility of normal tissue contamination.

We performed in vitro experiments to assess the vulnerabilities of cell lines with predicted druggable gene alterations to corresponding therapeutic agents. We tested six chemotherapeutic agents and five targeted therapeutic agents on eight cell lines with different predictive vulnerabilities (Supplementary Fig. 3; Supplementary Data 7) and the results were in line with the predictions (Fig. 4f). For chemotherapeutic agents, cells with oncogenic mutation in NF1 were significantly more sensitive to Vinblastine and cells with oncogenic mutations in ATM and ERCC4 were slightly more sensitive to Cisplatin than those without corresponding mutations. For targeted therapeutic agents, cells with CCNE1 amplifications were highly responsive to CDK2 inhibitor Roniciclib. Cells with oncogenic mutations in DNA repair genes BRCA2, ATM, ATR, CHEK2, and FANCA were more sensitive to PARP inhibitor Olaparib and cells with oncogenic mutations in TP53 or BRCA1 were more sensitive to WEE1 inhibitor MK-1775 than those without corresponding mutations. Cells with $C D K 4 / 6$ or FGF3/4 amplifications showed only slightly but significantly higher sensitivity to CDK4/6 inhibitor LEE011 or FGFR inhibitor Dovitinib, respectively, than those without corresponding gene amplifications (Fig. 4f).

Featured genomic and transcriptomic changes are correlated with survival in patients. Since tumor genomic and transcriptomic alterations may determine the clinical outcomes of patients, we analyzed the connections between key genomic and transcriptomic changes of ACGEJ and survival time in patients (see Methods). Regarding genomic changes, both TMB and gene level CNVs were significantly correlated with survival time: patients with low TMB $(<3.45$ per Mb) or more gene level CNVs $(\geq 382)$ in ACGEJ had short survival time (both log-rank $P=$ $0.030)$ compared with patients with high TMB $(\geq 3.45$ per $\mathrm{Mb})$ or less gene level CNVs $(<382)$ in ACGEJ, and the hazard ratios (HRs) of low TMB and more gene level CNVs for death adjusted for age, sex, and tumor stage were 7.70 (95\% confidence interval $(\mathrm{CI})=1.02-58.26)$ and $2.79(95 \% \mathrm{CI}=1.05-7.42)$, respectively (Fig. 5a, b). Given that the TMB and gene level CNVs were moderately correlated in our patients (Spearman's $\rho=0.32, P=$ $2.50 \mathrm{e}-4)$, we further classified patients into two groups, the lowTMB high-CNV group $(n=31)$ versus others $(n=52)$, and the 

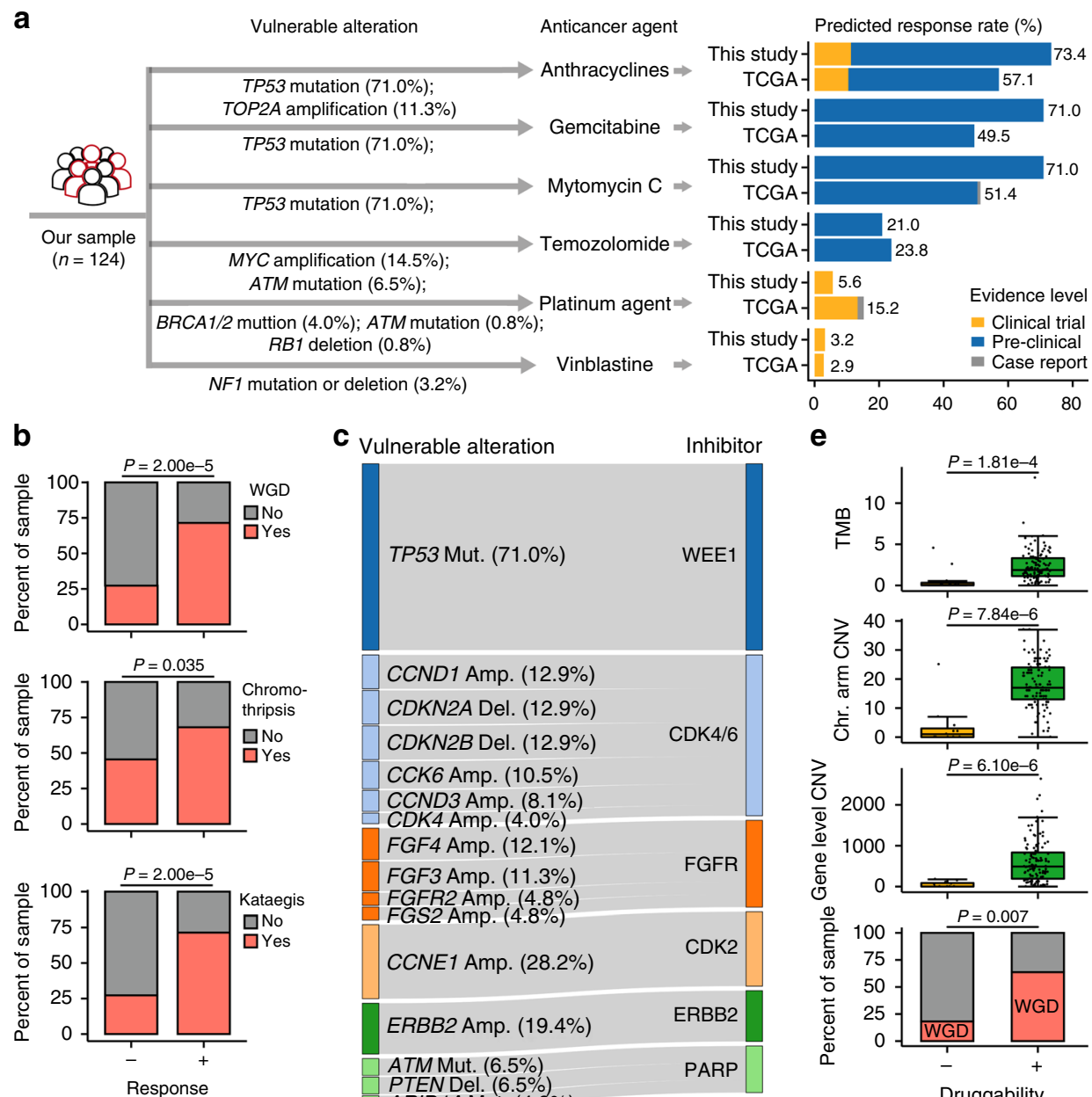

C Vulnerable alteration
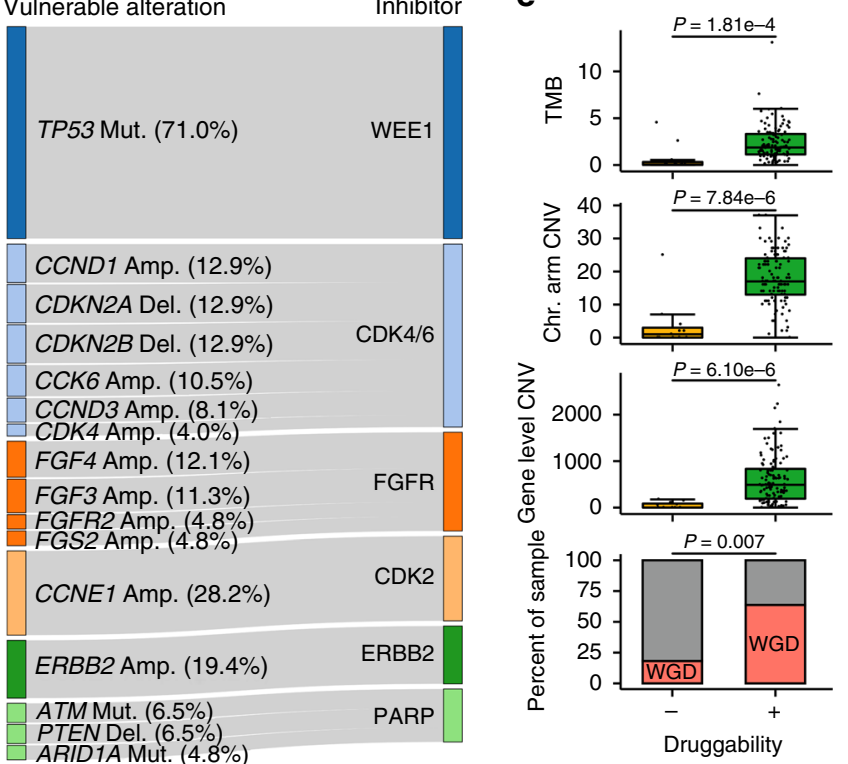

d
o
$\frac{0}{\pi}$
$\frac{0}{0}$
0
$\overline{0}$
$\frac{0}{0}$
$\frac{2}{0}$
$\frac{0}{0}$
$\frac{0}{0}$
$\frac{0}{2}$
0

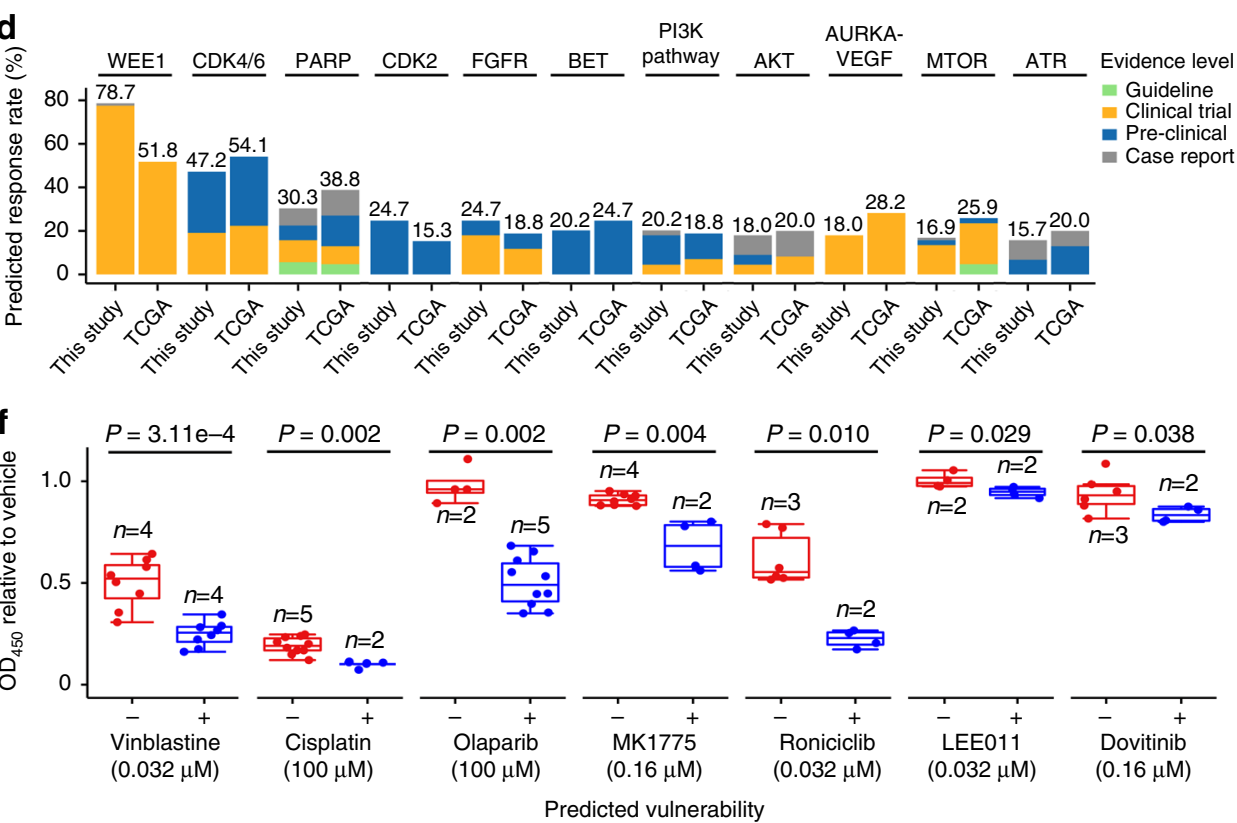

first group showed significantly shorter survival time than the second group ( $\log$-rank $P=0.001)$, with an adjusted HR of 4.61 $(95 \% \mathrm{CI}=1.80-11.80)$ (Fig. $5 \mathrm{c}$ ). Incorporating TMB with gene level CNVs led to a bigger HR than considering the latter alone, suggesting the two genomic alterations may combinedly affect ACGEJ survival time. We also found a significant correlation between Signature 17 activities $(\geq 17.1 \%$ or $<17.1 \%)$ in ACGEJ and survival time in patients (log-rank $P=0.022$; Fig. $5 \mathrm{~d}$ ). The HR of high Signature 17 activity for ACGEJ death was 3.94 (95\% $\mathrm{CI}=1.38-11.28)$ adjusted for age, sex, tumor stage, TMB, and gene level CNVs, indicating Signature 17 activity is an independent prognostic factor. Figure 5e shows a multivariate Cox model comparing the relative impacts of featured genomic alterations and other non-genomic factors such as for age, sex, and tumor 
Fig. 4 Genomic alterations vulnerable to existing treatment options in Chinese ACGEJ in comparison with TCGA ACGEJ. a Chemotherapeutic agents predicted to be responsive in Chinese ACGEJ. Left panel shows genomic alterations vulnerable to each chemotherapeutic agent and right panel shows the percentage of samples responsive to each chemotherapeutic agent, with comparisons between our ACGEJ patient set and the TCGA ACGEJ dataset. b Bar plots comparing ACGEJ samples likely responsive and unresponsive to Anthracyclines, Gemcitabine, or Mytomycin C ( $n=91$ and 33 , respectively) for the frequencies of WGD, chromothripsis, and kataegis (Fisher's exact tests). c Genomic alterations in our ACGEJ samples and the corresponding targeted therapeutic agents. Only genomic alterations detected in $\geq 5$ samples and agents having potential targets in $\geq 20 \%$ samples are shown. $\mathbf{d}$ Percentage of samples predicted to be responsive to different targeted therapeutic agents, with comparisons between our patients and TCGA patients without ERBB2 amplification ( $n=89$ and 85 , respectively). Only agents with potential targets in $\geq 20 \%$ of our patients or TCGA patients are shown. e Comparison between likely druggable and undruggable ACGEJ samples ( $n=113$ and 11, respectively) for TMB, chromosomal (Chr.) arm or gene level CNVs (two-sided Wilcoxon rank-sum tests), and the frequencies of WGD (Fisher's exact test). $\mathbf{f}$ In vitro validation of predicted vulnerabilities to chemotherapeutic and targeted therapeutic agents. Box plots compare relative viability (expressed as $\mathrm{OD}_{450}$ value) of different cell lines, likely vulnerable (blue) or invulnerable (red) to specified therapeutic agents, after treatment with optimal dose (in parentheses) of corresponding agents for $72 \mathrm{~h}$. Each dot represents the result of one independent experiment and each experiment had three replicates on one cell line; $n$ represents the number of cell lines; $P$ values derived from twosided Wilcoxon rank-sum tests. Box plots in (e, f) show the median (central line), the $25-75 \%$ interquartile range (IQR) (box limits), the \pm 1.5 times IQR (Tukey whiskers), and all data points, among which the lowest and the highest points indicate minimal and maximal values, respectively.

stage. We next evaluated the survival associations of ACGEJ transcriptomic changes, specifically, aberrant activities of cancer hallmark pathways or gene signatures quantified by GSVA scores. Patients with low GSVA scores of the IFN- $\alpha$ response pathway $(<-0.2256)$ showed significantly shorter survival time (log-rank $P=0.004$; Fig. 5f) than those with high scores $(\geq-0.2256)$. The HR of high IFN- $\alpha$ response for ACGEJ death was 0.25 (95\% CI = 0.09-0.66) adjusted for age, sex, and tumor stage. Because downregulated IFN- $\alpha$ response pathway was significantly correlated with high Signature 17 activities (Fig. 2e), we then focused analysis on this pathway and further identified two relevant genes, Interferon Induced Protein 44 (IFI44) and IFI30 Lysosomal Thiol Reductase (IFI30), whose high expressions ( $\geq 3.22$ or $\geq 28.13$ Transcripts Per Million, TPM) were significantly correlated with long survival time (log-rank $P=6.57 \mathrm{e}-5$ and 0.043 , respectively; Fig. $5 \mathrm{~g}, \mathrm{~h})$, with respective adjusted HRs of $0.23(95 \% \mathrm{CI}=$ $0.09-0.60)$ and 0.28 (95\% CI $=0.09-0.84)$.

We then investigated the survival associations of these genomic/transcriptomic alterations in TCGA patients with ACGEJ or non-ACGEJ CIN-type gastric cancer $(n=184)$ in the same way (see Methods). The results showed that TCGA patients with low TMB $(<1.73$ per $\mathrm{Mb})$ or high Signature 17 activities $(\geq 48.5 \%)$ had significantly shorter survival time (logrank $P=0.039$ and 0.034 , respectively) than those with high $\mathrm{TMB}(\geq 1.73$ per $\mathrm{Mb})$ or low Signature 17 activities $(<48.5 \%)$; the HRs of low TMB and high Signature 17 activity being 1.78 (95\% $\mathrm{CI}=0.98-3.22)$ and $2.61(95 \% \mathrm{CI}=1.26-5.38)$, respectively (Supplementary Fig. 4a, b). Patients with high IFN-a response $(\geq-0.4892)$ or high IFI30 expression $(\geq 10.80$ TPM) in tumors had significantly long survival time (log-rank $P=0.019$ and 0.020 , respectively), with the HRs of 0.46 (95\% CI $=0.27-0.79)$ and $0.51(95 \% \mathrm{CI}=0.28-0.93)$, respectively (Supplementary Fig. 4c, d). These results were consistent with the findings in our patient set. However, in TCGA patients, few gene level CNVs $(<42)$ and high IFI44 expression $(\geq 12.09$ TPM $)$ were associated with short survival time (log-rank $P=0.026$ and 0.023$)$, with the HRs of $2.45(95 \% \quad \mathrm{CI}=1.21-4.97)$ and $1.76 \quad(95 \% \quad \mathrm{CI}=$ 1.07-2.88), respectively (Supplementary Fig. 4e, f), which were inconsistent with the results in our patients. We also assessed the survival associations of IFI44 and IFI30 expressions using the gene expression data of gastric cancer patients registered in the Gene Expression Omnibus (GEO). Low IFI44 expression was consistently and significantly correlated with short survival time (log-rank $P=0.004, \mathrm{HR}=0.75,95 \% \mathrm{CI}=0.61-0.91$; Supplementary Fig. 4g), while low IFI30 expression was marginally correlated with short survival time (log-rank $P=$ $0.055, \mathrm{HR}=0.84,95 \% \mathrm{CI}=0.71-1.00$; Supplementary Fig. $4 \mathrm{~h}$ ).

\section{Discussion}

ACGEJ is on the rise worldwide, but its molecular characteristics have never been independently profiled and reported, which is impeding clinical treatment and drug development of this malignancy. In the present study, we have assembled and comprehensively analyzed the genome and transcriptome data of totally 124 ACGEJ samples exclusively collected from Chinese patients. The results have indicated that, like Caucasian patients, the ACGEJ genomes of our patients were dominated by CINpromoted tumorigenic focal CNVs while lacking recurrently mutated coding genes other than TP53. Both TMB and gene level CNVs of the ACGEJ genome indicate the prognosis of Chinese patients. Compared with previous reports, the present study has two major advances. Firstly, our integrative analyses on genome and bulk-tissue transcriptome sequencing data have led us to more reliable findings. Secondly, we have gone beyond descriptive genomic profiling to predict effective therapeutic agents and prognosis of patients based on their genomic and transcriptomic changes. The comprehensive findings in the present study may improve our current knowledge of ACGEJ and have implications for clinical care of patients.

In the ACGEJ genomes of our patients, TP53 is the only significantly and recurrently mutated coding gene we have detected. That said, there may be mutated non-coding drivers, such as CTCF-binding-site SNVs attributable to COSMIC Signature 17. As one of the hallmark signatures of upper-gastrointestinal adenocarcinoma, Signature 17 is believed to arise from misincorporation of oxidized DNA precursors into genomic $\mathrm{DNA}^{12,35}$. It has been shown that oxidative stress may generate more mutations in introns and intergenic regions than in exons and promoters $^{36}$, and improperly handled oxidative DNA damages can lead to CIN phenotypes in model systems ${ }^{37,38}$ and in human cells ${ }^{39}$. Our results are in line with these previous reports, indicating that Signature 17 SNVs may be attributed to oxidative stress and represents an etiological factor forming CIN in ACGEJ genomes. In addition, we have notably provided bulk transcriptomic evidence to support the role of Signature 17 by correlating its activities with a gene expression signature of CIN. More importantly, our analysis on bulk RNA sequencing data has revealed an immunosuppressive microenvironment in tumors with high activities of Signature 17. These results indicate that Signature 17 related genome and transcriptome changes play very important roles in the development and progression of ACGEJ, which may be of clinical relevance.

Comparing the genomic and transcriptomic alterations in our samples with those in TCGA ACGEJ samples (mostly from Caucasian patients) revealed similar CNV patterns and pathway aberrations. However, tumor samples of our patients seem to 
a

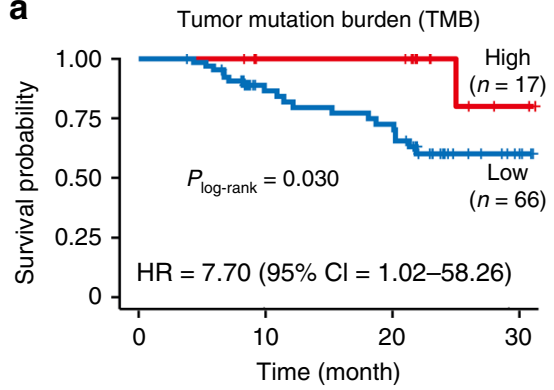

C

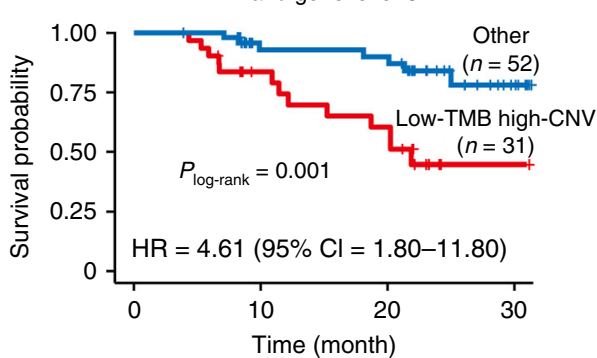

e

\begin{tabular}{lcc}
\hline Variable & No. of patient & $\mathrm{HR}(95 \% \mathrm{Cl})$ \\
\hline Age & 83 & $1.02(0.96-1.09)$ \\
Sex & & Reference \\
$\quad$ Female & 17 & $3.75(0.83-16.98)$ \\
Male & 66 & Reference \\
Stage & & $1.48(0.54-4.04)$ \\
I/II & 37 & $0.65(0.43-0.99)$ \\
III/IV & 46 & Reference \\
TMB & 83 & $4.00(1.37-11.65)$ \\
Gene level CNV & & \\
Low & 41 & Reference \\
High & 42 & $3.37(1.18-9.62)$ \\
Signature 17 activity & & \\
$\quad$ Low & 44 & \\
High & 39 &
\end{tabular}

g

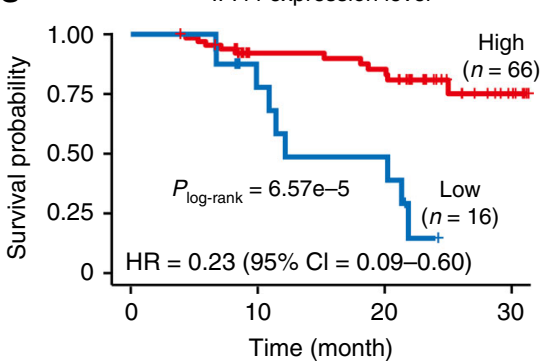

b

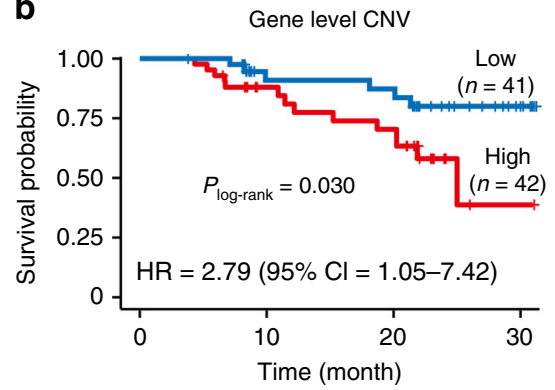

d

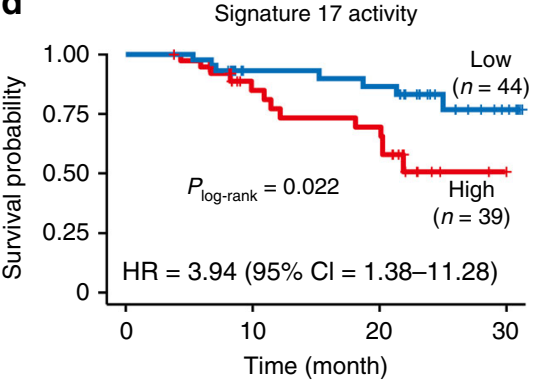

$\mathbf{f}$

IFN- $\alpha$ response pathway

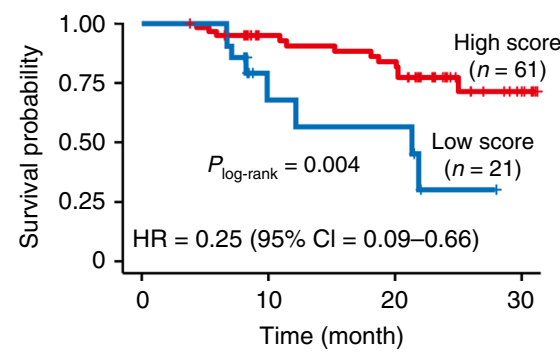

h

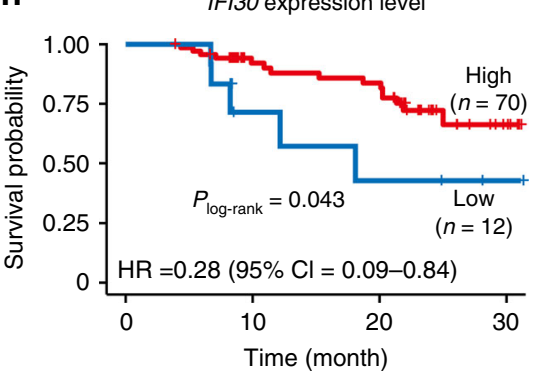

Fig. 5 Survival-associated genomic and transcriptomic features of ACGEJ. a-d Kaplan-Meier (KM) curves of patient survival according to TMB (a), gene level CNVs (b), TMB and gene level CNVs combined (c), and Signature 17 activities (d); $P$ values derived from log-rank tests. e Multivariate Cox regression analysis of the effects of different genomic alterations on ACGEJ patient survival. $\mathbf{f}-\mathbf{h}$ KM curves of patient survival according to the GSVA scores of IFN- $\alpha$ response pathway (f) and the expression levels of IFI44 (g) or IFI30 (h) gene; $P$ values derived from log-rank tests. Also present with each KM plot are the hazard ratio $(\mathrm{HR})$ and $95 \%$ confidence interval $(\mathrm{Cl})$ from multivariate Cox proportional hazard models including age, sex, tumor stage, $\mathrm{TMB}$, and gene level CNVs as covariates wherever necessary.

have fewer functional mutations than TCGA samples, which might reflect different genetic and environmental backgrounds between Chinese patients and Caucasian patients or might be simply due to different coverage of our WGS data and TCGA whole-exome sequencing data. We could reduce the confounding effect of different sequencing coverage by down-sampling the reads, but that requires access to controlled TCGA data. There are also some differences in the alteration rates of candidate driver genes (e.g., LIPF), potential drug targets (e.g., CCNE1), and survival-associated immune pathways (e.g., IFN- $\alpha$ response) across two cohorts. In these regards, the current study has increased general knowledge on the genetic and molecular basis of ACGEJ and, particularly, filled in the gap for non-Caucasian patients. Thus, our findings could contribute to developing more specific and effective precise care of ACGEJ worldwide.

Because there have been no specific and effective drugs for ACGEJ treatments, we have screened the ACGEJ genome and transcriptome of each patient for changes that may indicate the patient's responses to therapeutic agents currently used for other types of cancer or under clinical trials. Since DNA repair 
deficiency caused by TP53 inactivation and other events is the major mechanism associated with the efficacy of cytotoxic anticancer drugs, we have predicted that more frequent TP53 inactivating mutations may lead to generally higher response rates to cytotoxic anticancer agents in Chinese patients than in Caucasian patients, suggesting that these chemotherapeutic agents may generally benefit Chinese patients with ACGEJ. We have also found that a considerable proportion of Chinese ACGEJ patients might benefit from targeted anticancer agents ${ }^{40}$ such as ERBB2, WEE1, and CDK4/6 inhibitors. The predicted response rates to these agents are somewhat different between Chinese and Caucasian ACGEJ patients. Specifically, we have shown that CCNE1 amplification is a CIN-associated oncogenic event prevalent in Chinese patients and often coexisting with ERBB2 amplifications. Given that CCNE1 amplification has been linked to the resistance to $E R B B 2$ inhibitors ${ }^{41,42}$, it is worthwhile to conduct clinical trials for CDK2 inhibitors ${ }^{43,44}$ in Chinese ACGEJ patients. We have validated the reliability of gene-alteration-based drug vulnerability prediction by in vitro assays in a panel of cancer cell lines. Thus, our predictions on the response rates to currently used cytotoxic anticancer agents or targeted drugs approved or tested for other types of cancer have provided molecular evidence for their clinical use or clinical trials for treating ACGEJ.

In the present study, we have identified several prognostic markers from genomic or transcriptomic changes associated with the survival time in ACGEJ patients. At the genomic level, we have found that TMB and gene level CNVs are independently and combinedly correlated with the survival time in our patients. In addition, we have identified Signature 17 activity as another independent marker for ACGEJ survival time, which one may expect because in our patient set Signature 17 is correlated with CIN-related genomic changes such as WGD and chromothripsis, both confer terrible malignant cancer phenotypes ${ }^{23,45}$. Consistent with our result, a previous genomic study in breast cancer has also shown that Signature 17 is enriched in metastatic tumors and linked to poor prognosis of patients ${ }^{46}$. Nevertheless, in the present study we went beyond genome data and incorporated transcriptome data for analysis. As a result, we have found that a Signature 17-correlated cancer hallmark pathway, IFN- $\alpha$ response, is also significantly associated with the survival time in our ACGEJ patient set. Moreover, transcriptome analysis has shown that tumors with high Signature 17 activities had repressed activities of IFN-producing cytotoxic cells. These findings emphasize that immunosuppression in the tumor microenvironment, likely induced by high Signature 17 activities in cancer cells, plays an important role in the progression of ACGEJ. Specifically, we have found significant associations of high expression levels of IFI44 and IFI30, two IFN- $\alpha$-inducible genes, with good survival in our patient set. Our comparative analyses on TCGA and GEO datasets have yielded both consistent and inconsistent results. The associations of TMB levels, Signature 17 activities, IFN- $\alpha$ response activities, and IFI30 expression with patient survival time are quite consistent in our patient set and TCGA and GEO patient set. The results for gene level CNVs in our cohort and the TCGA cohort seem contradictory. For IFI44, the GEO data agreed with our finding, whereas the TCGA data showed a significantly negative correlation between high IFI44 expression and patient survival. IFI44 has been reported to function in many biological processes through IFN signaling pathways, including anti-proliferative activity in tumors ${ }^{47,48}$. High IFI30 expression in tumor cells has been linked to improved cancer survival ${ }^{49,50}$, possibly due to its ability to enhance the presentation of tumor antigens for $\mathrm{T}$ cell recognition ${ }^{51}$ or to regulate the cellular redox state and proliferation ${ }^{52}$. Thus, the identified down-regulation of IFI44 and IFI30 might be a simplified prognostic marker for ACGEJ in clinical use.
Despite the advances discussed above, we acknowledge some limitations in the present study. Firstly, we focused on genomic alterations in coding sequences and barely touched upon noncoding elements, which may harbor additional ACGEJ drivers. Secondly, we have not fully investigated the extent of intratumor heterogeneity and how it may affect our findings. Both issues are on our agenda for future studies. In addition, the clinical or preclinical evidence for the anticancer agents we referred to mostly came from studies on Caucasian patients and thus it is uncertain whether and how much they will apply to Chinese patients.

In conclusion, our present study has profiled ACGEJ in Chinese patients as a CNV-dominant CIN-type tumor, revealed the types and the distributions of various druggable changes in tumor genomes, and identified genomic and transcriptomic prognostic markers that have potential clinical implications. These findings have furthered our understanding on ACGEJ and would help develop more effective therapeutic strategies to precisely fight this malignancy, especially for Chinese patients.

\section{Methods}

Biospecimen and clinical data. The biospecimen used in this study were obtained from 124 Chinese ACGEJ patients (Supplementary Table 3) recruited at the Linzhou Cancer Hospital and Linzhou Esophageal Cancer Hospital (Henan Province, China) between 2013 and 2018. ACGEJ tumor, adjacent non-tumor gastric tissue ( $\geq 5 \mathrm{~cm}$ from tumor margin), and peripheral blood samples were collected at the time of surgical resection. ACGEJ (tumors arising at the gastric cardia and/or gastroesophageal junction with/without involvement of other esophageal and/or gastric subsites) was confirmed by two pathologists via histopathological examination. No patient had received chemotherapy or radiotherapy before surgery. Clinical data were collected from the medical record of each patient. We conducted follow-up phone interviews with 83 patients in a period of 20.2 months (median). At the most recent interview (November 2018), 75.9\% (63/83) of patients were still alive. This study was approved by the Institutional Review Board of Cancer Hospital, Chinese Academy of Medical Sciences. Written informed consent was solicited from every patient prior to sample collection.

DNA and RNA sequencing. Only tumor samples containing $\geq 60 \%$ of cancer cells evaluated by examining tissue slides (Supplementary Fig. 1a) were used for DNA and RNA sequencing. WGS data were generated from matched tumor and blood samples from 124 patients. The bulk RNA sequencing data were generated from matched tumor and normal tissue samples from 123 of the 124 patients. DNA and total RNA were extracted from tissue samples using the AllPrep DNA/RNA Kit (Qiagen). Blood DNA was extracted using QiaAmp Blood Midi Kit (Qiagen). Library preparation was as previously described ${ }^{53}$. All libraries were sequenced on Illumina HiSeq xTen in $2 \times 150$ bp paired-end mode.

Detection of somatic SNVs, indels, CNVs, and SVs. DNA sequence reads were aligned to the Ensembl GRCh37 human genome using BWA-MEM (v0.1.22) ${ }^{54}$. Somatic mutations (SNVs and indels) were detected using Strelka2 (v2.8.3) ${ }^{55}$ and annotated by Ensembl Variant Effect Predictor (VEP, release 90) ${ }^{56}$. We filtered the results with gatk-tools (v0.2.2). TMB was measured by the number of non-silent SNVs/indels. Somatic CNVs (based on the log ratio of tumor to non-tumor reads) were detected from WGS data by BIC-seq2 (v0.2.4) ${ }^{57}$. Allelic copy number, ploidy, and purity were estimated by FACETS (v0.5.14) ${ }^{58}$. Large-scale ( $>50 \mathrm{bps}$ ) SVs were independently called from the WGS data by Delly (v0.7.3) ${ }^{59}$, GRIDSS (v2.4.0 ${ }^{60}$, Manta (v1.1.1) ${ }^{61}$, and svABA (v0.2.1 ${ }^{62}$; then we used SURVIVOR (v1.0.6) ${ }^{63}$ to merge nearby break points and filter out SVs detected by only one tool.

Identification of specific genomic features. We used MutSigCV (v1.41) ${ }^{64}$ to identify genes with significantly recurrent coding-sequence SNVs/indels and ActiveDriverWGS (v1.0.1) ${ }^{65}$ to identify non-coding genomic elements (e.g., promoters, non-coding RNAs) with significantly more unexpected somatic SNVs. These genes and genomic elements were deemed potential drivers of ACGEJ. Functional mutations were defined as SNVs/indels likely affecting the function of a gene, including in-frame and frame-shift indels, nonsense SNVs, OncoKB annotated oncogenic or likely oncogenic missense SNVs, or SNVs/indels occurring on splice site, start codon, or stop codon.

Segmentation files output by BIC-seq2 (for our samples) or downloaded from the TCGA website (excluding the regions within $3 \mathrm{Mb}$ around centromeres and $1 \mathrm{Mb}$ at both chromosome ends) were used as input for GSITIC2.0 (v.2.0.23) ${ }^{66}$ to quantify the CNV status of each gene in each tumor genome. In a tumor genome, we considered a gene undergoing homozygous deletion, copy-number loss, copynumber gain, or amplification if the corresponding GISTIC score $=-2, \leq-1, \geq 1$, or $=2$, respectively. Significantly recurrent focal CNV regions (Supplementary Data 8,9 ) were also determined by GISTIC2.0 (FDR $q<0.25)$. 
We considered a chromosomal arm undergoing copy-number gain or loss if $\geq 2 / 3$ genes on that arm had GISTIC score $\geq 1$ or $\leq-1$, respectively. We defined arm level CNVs as chromosomal arms with copy-number gain or loss and gene level CNVs as gene amplifications or deletions. Only genes with GISTIC scores \pm 2 were counted when measuring gene level CNVs. We considered an ACGEJ genome undergoing WGD if $\geq 50 \%$ autosomes had a major allele copy number $\geq 2$; the $50 \%$ threshold was determined following a previous study ${ }^{23}$ (Supplementary Fig. 5). We measured the proportion of CNV affected genome using regions where $\mid \log 2$ (reads ratio) $\mid \geq 0.3$. Kataegis events were detected following the method of a previous WGS study ${ }^{67}$. Chromothripsis events were identified by ShatterSeek (v0.4) ${ }^{45}$ based on the merged SV results and FACETS CNV results.

Identification of mutational signatures. We started by extracting de novo signatures from and inferring the prevalence of 30 published COSMIC mutational signatures in the Chinese cohort. Both had led us to recognize the prevalent activities of COSMIC Signatures 1, 3, 5, 8, and 17 (or their analogues). Thus, we only focused on these five signatures and clamped the activities of other signatures to zero in all subsequent analyses. We used deconstructSigs (v1.8.0) ${ }^{68}$ to measure the activity of every signature in every tumor genome (the percentage of SNVs across the exome or genome attributable to the signature). For each somatic SNV, we determined its clonality and the mutational signature it most likely belongs to using Palimpsest (v.2.0.0 $)^{69}$.

Analysis of bulk-tissue RNA sequencing data. We used HISAT2 (v2.1.0 $)^{70}$ to align RNA sequence reads to the Ensembl GRCh37 human genome and then used StringTie (v1.3.3b) ${ }^{71}$ to re-assemble transcriptome and quantify the expression level of each gene in each sample. The assembly process was guided by the reference annotations from GENCODE (v19) ${ }^{72}$. Gene expression levels were quantified by Transcript per Million (TPM). We considered a gene differentially expressed in tumor and normal samples if the $\log 2+1$ transformed TPM change was significant in a paired Student's $t$-test (FDR $q<0.05)$ and the fold change of mean TPM was $>1.2$ or $<0.8$. In other comparing situations, we considered a gene differentially expressed in two groups of samples if the TPM change was significant in a paired Wilcoxon test $(P<0.05)$ and the fold change of median TPM was $>1.2$ or $<0.8$. The activity of specific pathway or gene signature in each tumor or normal sample was quantified using the R package GSVA (v1.30.0) ${ }^{31}$ on $\log 2+1$ transformed TPM. The differential expression of specific pathway or gene signature between tumor and normal samples was assessed using gene set enrichment analysis (GSEA) ${ }^{73}$ (implemented in the R package clusterProfiler v3.10.1 ${ }^{74}$ ). We quantified the fractions of 10 immune cell types (i.e., macrophages (M1 and M2), monocytes, neutrophils, B, NK, CD8 + /CD4 +/regulatory T, myeloid dendritic cells, and uncharacterized cells) in individual tumor and adjacent normal samples using quanTIseq ${ }^{75}$ (implemented in the R package immunedeconv v2.0.0)

Identification of Signature17-correlated pathways and gene signatures. We identified these pathways and gene signatures using matched WGS and bulk RNAseq data generated from 123 patients. We first calculated partial Spearman's correlation (using the R package ppcor v1.1) between Signature 17 activities and the expressions of each gene across tumor samples, controlling for tumor purity and the activities of other four signatures (i.e., Signatures 1, 3, 5, and 8). Then we examined known cancer hallmark pathways and gene signatures obtained from a previous study ${ }^{30}$ one by one to see whether they are enriched with Signature 17correlated genes using GSEA on the calculated correlation coefficients.

Analysis and integration of public data. To fairly compare the transcriptomic profiles of our ACGEJ samples and TCGA samples, we combined the gene expression data (measured by TPM) of both cohorts and that of normal gastroesophageal junction tissue samples from the Genotype-Tissue Expression (GTEx) Project, performed normalization and batch correction and used the processed data for comparative analyses. We normalized the combined gene expression dataset by keeping genes whose expression levels were quantified in all three individual datasets $(n=19,587)$ and rescaling the TPM values per sample to maintain a sum of 1 million. The ComBat function implemented in the R package sva (v3.28.0) ${ }^{76}$ was used to remove the batch effect of different data sources (i.e., data source as a batch parameter). Tumor and normal samples in the combined dataset were respectively grouped together and appropriately separated from each other (Supplementary Fig. 6). The GSVA scores of featured pathways and gene signatures in either cohort before and after the processing were highly correlated; so were the estimated fractions of different immune cell types.

In vitro validation of drug vulnerability prediction. We used eight human cancer cell lines including the ACGEJ cell line OE19 (purchased from Beijing Beina Chuanglian Biotechnology Institute), esophageal adenocarcinoma cell lines OE33 and SK-GT-4 (purchased from Nanjing COBIOER Biosciences Company Limited), gastric adenocarcinoma cell lines AGS and HGC-27, and colorectal cancer cell lines HCT-116, LoVo and RKO (purchased from the Cell Bank of Type Culture Collection of Chinese Academy of Sciences Shanghai Institute of Biochemistry and Cell Biology). We obtained somatic mutation and CNV data of these cell lines from COSMIC Cell Lines Project (https://cancer.sanger.ac.uk/cell_lines) and predicted each cell line as vulnerable or invulnerable to specific chemotherapeutic or targeted therapeutic agents based on whether it carried corresponding gene alterations. The efficacy of 11 drugs was respectively assessed by cell viability measured using the CCK-8 kit (Dojindo Labs). Briefly, cells were treated with optimal dose of each drug and the viability was measured after incubation for $72 \mathrm{~h}$. All analyses were performed in two independent experiments and each had three replicates.

Survival analysis. We used the log-rank test in univariate survival analyses and the Cox proportional hazards model in multivariate survival analyses (both implemented in the R package survival v2.43-3). The Kaplan-Meier plot was used for presentation. The specific cutoff we used to dichotomize a continuous variable (e.g., gene level CNVs, Signature 17 activities, IFI44 expression) and then group patients was determined by testing a series of values of that variable with fixed increments and then choosing the one by which both log-rank and Cox $P$ were $<0.05$ and the $\log$-rank $P$ was minimized. This process would not give us any options if there was no statistically significant result in the first place. The cutoffs we used to obtain the presented results were 3.45 per $\mathrm{Mb}$ for TMB, 382 for gene level CNVs, $17.1 \%$ for Signature 17 activities, -0.2256 for IFN- $\alpha$ response, 3.22 for IFI44 expressions, and 28.13 for IFI30 expressions. The same approach was applied to divide TCGA patients into good or poor survival groups, with the specific cutoffs of 1.73 per $\mathrm{Mb}$ for TMB, 42 for gene level CNVs, $48.5 \%$ for Signature 17 activity, -0.4892 for IFNa response, 12.09 for IFI44 expressions, and 10.80 for IFI33 expressions.

Identification of prognostic marker genes. To identify prognostic marker genes in the IFN- $\alpha$ response pathway, we first selected 40 genes whose expressions were (a) significantly correlated with the pathway GSVA scores across tumor samples (| Spearman's $\rho \mid>0.3, P<0.05)$ and (b) significantly different between the two survival groups divided based on their IFN- $\alpha$ pathway GSVA scores $(t$-test $P<0.05)$. Next, we tested each of the 40 genes and found 13 of them were significantly correlated with patient survival (with at least one cutoff under which log-rank and Cox $P<0.05$ ). We finally picked IFI44 and IFI30 out of the 13 genes using a LassoCox method as implemented in the glmnet package v3.0-2

Statistical analysis. We used Fisher's exact test for any independence test between two categorical variables and Wilcoxon rank-sum test for any independence test between a continuous variable and a binary categorical variable, when there was no covariate to adjust for. Otherwise, we used an F-test to compare two generalized linear models, one of which included the variable being evaluated as a predictor. Spearman's rank correlation coefficient was used to measure the correlation between two continuous variables.

Reporting summary. Further information on research design is available in the Nature Research Reporting Summary linked to this article.

\section{Data availability}

The raw WGS and RNA sequencing data generated in this study are deposited in the Genome Sequence Archive of Beijing Institute of Genomics, Chinese Academy of Science (http://bigd.big.ac.cn/gsa/, accession number HRA000025). The gene expression data are also publicly available from NCBI Gene Expression Omnibus (https://www.ncbi.nlm.nih gov/geo, accession number GSE159721). The whole-genome somatic variants are also publicly available from the European Variation Archive (https://www.ebi.ac.uk/eva, accession number PRJEB41070). We obtained somatic mutation and CNV data of TCGA ACGEJ samples ${ }^{8}$ from the Broad Institute GDAC Firehose website (https://gdac. broadinstitute.org/) and somatic mutation data of additional 46 ACGEJ samples ${ }^{15,16}$ from the Tumor Portal (http://www.tumorportal.org). The gene expression (TPM) data of TCGA ACGEJ samples ${ }^{10}$ were obtained from TCGA Pan-cancer Atlas publication web page (https://gdc.cancer.gov/about-data/publications/pancanatlas). The gene expression (TPM) data of normal gastroesophageal junction tissue samples ${ }^{78}$ were obtained from the GTEx Portal (http://www.gtexportal.org/home/). Additional survival analyses in the GEO datasets were conducted on the Kaplan-Meier Plotter website (https://kmplot.com) ${ }^{79}$ with automatically selected best cutoffs. Other data that support the findings of this study are available within the supplementary files or available from the authors upon request.

\section{Code availability}

We used published software for all our analyses as indicated. Other accompanying code is available from the authors upon request.

Received: 10 April 2020; Accepted: 8 November 2020; Published online: 30 November 2020

\section{References}

1. Buas, M. F. \& Vaughan, T. L. Epidemiology and risk factors for gastroesophageal junction tumors: understanding the rising incidence of this disease. Semin. Radiat. Oncol. 23, 3-9 (2013) . 
2. Liang, D. et al. Gastric cancer burden of last 40 years in north china (hebei province): a population-based study. Medicine 96, e5887 (2017).

3. Ajani, J. A. et al. Esophageal and esophagogastric junction cancers, version 2.2019, NCCN clinical practice guidelines in oncology. J. Natl. Compr. Cancer Netw. 17, 855-883 (2019).

4. Lin, Y. et al. Epidemiology of esophageal cancer in Japan and China. J. Epidemiol. 23, 233-242 (2013).

5. Greally, M., Agarwal, R. \& Ilson, D. H. Optimal management of gastroesophageal junction cancer. Cancer 125, 1990-2001 (2019).

6. Fuchs, C. S. et al. Ramucirumab monotherapy for previously treated advanced gastric or gastro-oesophageal junction adenocarcinoma (REGARD): an international, randomised, multicentre, placebo-controlled, phase 3 trial. Lancet 383, 31-39 (2014).

7. Boku, N. et al. Safety and efficacy of nivolumab in combination with S-1/ capecitabine plus oxaliplatin in patients with previously untreated, unresectable, advanced, or recurrent gastric/gastroesophageal junction cancer: Interim results of a randomized, phase II trial (ATTRACTION-4). Ann. Oncol. 30, 250-258 (2019).

8. Cancer Genome Atlas Research Network. Comprehensive molecular characterization of gastric adenocarcinoma. Nature 513, 202-209 (2014).

9. Alexandrov, L. B. et al. Signatures of mutational processes in human cancer. Nature 500, 415-421 (2013).

10. Liu, Y. et al. Comparative molecular analysis of gastrointestinal adenocarcinomas. Cancer Cell 33, 721-735 (2018).

11. Gerstung, M. et al. The evolutionary history of 2,658 cancers. Nature 578, 122-128 (2020).

12. Nones, K. et al. Genomic catastrophes frequently arise in esophageal adenocarcinoma and drive tumorigenesis. Nat. Commun. 5, 1-9 (2014).

13. Cristescu, R. et al. Molecular analysis of gastric cancer identifies subtypes associated with distinct clinical outcomes. Nat. Med. 21, 449-456 (2015).

14. Xing, R. et al. Whole-genome sequencing reveals novel tandem-duplication hotspots and a prognostic mutational signature in gastric cancer. Nat. Commun. 10, 1-13 (2019).

15. Wang, K. et al. Exome sequencing identifies frequent mutation of ARID1A in molecular subtypes of gastric cancer. Nat. Genet. 43, 1219-1223 (2011).

16. Dulak, A. M. et al. Exome and whole-genome sequencing of esophageal adenocarcinoma identifies recurrent driver events and mutational complexity. Nat. Genet. 45, 478-486 (2013).

17. Sansregret, L., Vanhaesebroeck, B. \& Swanton, C. Determinants and clinical implications of chromosomal instability in cancer. Nat. Rev. Clin. Oncol. 15, 139-150 (2018).

18. Bakhoum, S. F. \& Cantley, L. C. The multifaceted role of chromosomal instability in cancer and its microenvironment. Cell 174, 1347-1360 (2018).

19. Carter, S. L., Eklund, A. C., Kohane, I. S., Harris, L. N. \& Szallasi, Z. A signature of chromosomal instability inferred from gene expression profiles predicts clinical outcome in multiple human cancers. Nat. Genet. 38, 1043-1048 (2006).

20. Dewhurst, S. M. et al. Tolerance of whole-genome doubling propagates chromosomal instability and accelerates cancer genome evolution. Cancer Disco. 4, 175-185 (2014)

21. Chakravarty, D. et al. OncoKB: a precision oncology knowledge base. JCO Precis. Oncol. 1, 1-16 (2017).

22. Chia, N. Y. et al. Regulatory crosstalk between lineage-survival oncogenes KLF5, GATA4 and GATA6 cooperatively promotes gastric cancer development. Gut 64, 707-719 (2015).

23. Bielski, C. M. et al. Genome doubling shapes the evolution and prognosis of advanced cancers. Nat. Genet. 50, 1189-1195 (2018).

24. Zack, T. I. et al. Pan-cancer patterns of somatic copy number alteration. Nat. Genet. 45, 1134-1140 (2013).

25. Spruck, C. H., Won, K. A. \& Reed, S. I. Deregulated cyclin E induces chromosome instability. Nature 401, 297-300 (1999).

26. Aziz, K. et al. Ccnel overexpression causes chromosome instability in liver cells and liver tumor development in mice. Gastroenterology 157, 210-226 (2019).

27. Katainen, R. et al. CTCF/cohesin-binding sites are frequently mutated in cancer. Nat. Genet. 47, 818-821 (2015).

28. Liu, E. M. et al. Identification of cancer drivers at CTCF insulators in 1,962 whole genomes. Cell Syst. 8, 446-455 (2019).

29. Guo, Y. A. et al. Mutation hotspots at CTCF binding sites coupled to chromosomal instability in gastrointestinal cancers. Nat. Commun. 9, 1-14 (2018).

30. Tamborero, D. et al. A pan-cancer landscape of interactions between solid tumors and infiltrating immune cell populations. Clin. Cancer Res. 24, 3717-3728 (2018).

31. Hanzelmann, S., Castelo, R. \& Guinney, J. GSVA: gene set variation analysis for microarray and RNA-seq data. Bioinformatics 14, 7 (2013).

32. Tamborero, D. et al. Cancer genome interpreter annotates the biological and clinical relevance of tumor alterations. Genome Med. 10, 25 (2018).
33. Wang, J. et al. TOP2A amplification in breast cancer is a predictive marker of anthracycline-based neoadjuvant chemotherapy efficacy. Breast Cancer Res. Treat. 135, 531-537 (2012).

34. Sohn, B. H. et al. Clinical significance of four molecular subtypes of gastric cancer identified by the cancer genome atlas project. Clin. Cancer Res. 23, 4441-4449 (2017).

35. Tomkova, M., Tomek, J., Kriaucionis, S. \& Schuster-Bockler, B. Mutational signature distribution varies with DNA replication timing and strand asymmetry. Genome Biol. 19, 1-12 (2018).

36. Poetsch, A. R., Boulton, S. J. \& Luscombe, N. M. Genomic landscape of oxidative DNA damage and repair reveals regioselective protection from mutagenesis. Genome Biol. 19, 1-23 (2018).

37. Degtyareva, N. P., Chen, L., Mieczkowski, P., Petes, T. D. \& Doetsch, P. W. Chronic oxidative DNA damage due to DNA repair defects causes chromosomal instability in saccharomyces cerevisiae. Mol. Cell Biol. 28, 5432-5445 (2008)

38. Thomson, G. J. et al. Metabolism-induced oxidative stress and DNA damage selectively trigger genome instability in polyploid fungal cells. EMBO J. $\mathbf{3 8}$, e101597 (2019).

39. Coluzzi, E. et al. Oxidative stress induces persistent telomeric DNA damage responsible for nuclear morphology change in mammalian cells. PLoS One $\mathbf{9}$, e110963 (2014)

40. Bedard, P. L., Hyman, D. M., Davids, M. S. \& Siu, L. L. Small molecules, big impact: 20 years of targeted therapy in oncology. Lancet 395, 1078-1088 (2020).

41. Kim, J. et al. Preexisting oncogenic events impact trastuzumab sensitivity in ERBB2-amplified gastroesophageal adenocarcinoma. J. Clin. Invest. 124, 5145-5158 (2014)

42. Janjigian, Y. Y. et al. Genetic predictors of response to systemic therapy in esophagogastric cancer. Cancer Disco. 8, 49-58 (2018).

43. Au-Yeung, G. et al. Selective targeting of cyclin E1-amplified high-grade serous ovarian cancer by cyclin-dependent kinase 2 and AKT inhibition. Clin. Cancer Res. 23, 1862-1874 (2017).

44. Chen, Z. et al. Characterization and validation of potential therapeutic targets based on the molecular signature of patient-derived xenografts in gastric cancer. J. Hematol. Oncol. 11, 20 (2018).

45. Cortes-Ciriano, I. et al. Comprehensive analysis of chromothripsis in 2,658 human cancers using whole-genome sequencing. Nat. Genet. 52, 331-341 (2020).

46. Bertucci, F. et al. Genomic characterization of metastatic breast cancers. Nature 569, 560-564 (2019).

47. Hallen, L. C. et al. Antiproliferative activity of the human IFN-alpha-inducible protein IFI44. J. Interferon Cytokine Res. 27, 675-680 (2007).

48. Wang, H. et al. Long Noncoding RNA LINC01116 contributes to gefitinib resistance in non-small cell lung cancer through regulating IFI44. Mol. Ther. Nucleic Acids 19, 218-227 (2020).

49. Xiang, Y. J. et al. Absence of gamma-interferon-inducible lysosomal thiol reductase (GILT) is associated with poor disease-free survival in breast cancer patients. PLoS One 9, e109449 (2014).

50. Rausch, M. P. \& Hastings, K. T. Diverse cellular and organismal functions of the lysosomal thiol reductase GILT. Mol. Immunol. 68, 124-128 (2015).

51. Rausch, M. P. et al. GILT accelerates autoimmunity to the melanoma antigen tyrosinase-related protein 1. J. Immunol. 185, 2828-2835 (2010).

52. Chiang, H. S. \& Maric, M. Lysosomal thiol reductase negatively regulates autophagy by altering glutathione synthesis and oxidation. Free Radic. Biol. Med. 51, 688-699 (2011).

53. Chang, J. et al. Genomic analysis of oesophageal squamous-cell carcinoma identifies alcohol drinking-related mutation signature and genomic alterations. Nat. Commun. 8, 1-11 (2017).

54. $\mathrm{Li}, \mathrm{H}$. Aligning sequence reads, clone sequences and assembly contigs with BWA-MEM. Preprint at http://arXiv:1303.3997 (2013).

55. Kim, S. et al. Strelka2: fast and accurate calling of germline and somatic variants. Nat. Methods 15, 591-594 (2018).

56. McLaren, W. et al. The ensembl variant effect predictor. Genome Biol. 17, 122 (2016).

57. Xi, R., Lee, S., Xia, Y., Kim, T.-M. \& Park, P. J. Copy number analysis of whole-genome data using BIC-seq2 and its application to detection of cancer susceptibility variants. Nucleic Acids Res. 44, 6274-6286 (2016).

58. Shen, R. \& Seshan, V. E. FACETS: allele-specific copy number and clonal heterogeneity analysis tool for high-throughput DNA sequencing. Nucleic Acids Res. 44, el31 (2016).

59. Rausch, T. et al. DELLY: structural variant discovery by integrated paired-end and split-read analysis. Bioinformatics 28, i333-i339 (2012).

60. Cameron, D. L. et al. GRIDSS: sensitive and specific genomic rearrangement detection using positional de Bruijn graph assembly. Genome Res. 27, 2050-2060 (2017) 
61. Chen, X. et al. Manta: rapid detection of structural variants and indels for germline and cancer sequencing applications. Bioinformatics 32, 1220-1222 (2015).

62. Wala, J. A. et al. SvABA: genome-wide detection of structural variants and indels by local assembly. Genome Res. 28, 581-591 (2018).

63. Jeffares, D. C. et al. Transient structural variations have strong effects on quantitative traits and reproductive isolation in fission yeast. Nat. Commun. 8 1-11 (2017).

64. Lawrence, M. S. et al. Mutational heterogeneity in cancer and the search for new cancer-associated genes. Nature 499, 214-218 (2013).

65. Zhu, H. et al. Candidate cancer driver mutations in distal regulatory elements and long-range chromatin interaction networks. Mol. Cell 77, 1307-1321 (2020).

66. Mermel, C. H. et al. GISTIC2.0 facilitates sensitive and confident localization of the targets of focal somatic copy-number alteration in human cancers. Genome Biol. 12, R41 (2011).

67. Secrier, M. et al. Mutational signatures in esophageal adenocarcinoma define etiologically distinct subgroups with therapeutic relevance. Nat. Genet. 48, 1131-1141 (2016).

68. Rosenthal, R., McGranahan, N., Herrero, J., Taylor, B. S. \& Swanton, C. Deconstructsigs: delineating mutational processes in single tumors distinguishes DNA repair deficiencies and patterns of carcinoma evolution. Genome Biol. 17, 31 (2016).

69. Letouze, E. et al. Mutational signatures reveal the dynamic interplay of risk factors and cellular processes during liver tumorigenesis. Nat. Commun. 8, 1-13 (2017).

70. Kim, D., Langmead, B. \& Salzberg, S. L. HISAT: a fast spliced aligner with low memory requirements. Nat. Methods 12, 357-360 (2015)

71. Pertea, M., Kim, D., Pertea, G. M., Leek, J. T. \& Salzberg, S. L. Transcript-level expression analysis of RNA-seq experiments with HISAT, StringTie and Ballgown. Nat. Protoc. 11, 1650-1667 (2016).

72. Harrow, J. et al. GENCODE: the reference human genome annotation for the ENCODE project. Genome Res. 22, 1760-1774 (2012).

73. Subramanian, A. et al. Gene set enrichment analysis: a knowledge-based approach for interpreting genome-wide expression profiles. Proc. Natl. Acad. Sci. USA 102, 15545-15550 (2005).

74. Yu, G., Wang, L. G., Han, Y. \& He, Q. Y. clusterProfiler: an R package for comparing biological themes among gene clusters. OMICS 16, 284-287 (2012).

75. Finotello, F. et al. Molecular and pharmacological modulators of the tumor immune contexture revealed by deconvolution of RNA-seq data. Genome Med. 11, 34-53 (2019).

76. Leek, J. T., Johnson, W. E., Parker, H. S., Jaffe, A. E. \& Storey, J. D. The sva package for removing batch effects and other unwanted variation in highthroughput experiments. Bioinformatics 28, 882-883 (2012).

77. Friedman, J., Hastie, T. \& Tibshirani, R. Regularization paths for generalized linear models via coordinate descent. J. Stat. Softw. 33, 1-22 (2010).

78. Aguet, F. et al. Genetic effects on gene expression across human tissues. Nature 550, 204-213 (2017).

79. Szasz, A. M. et al. Cross-validation of survival associated biomarkers in gastric cancer using transcriptomic data of 1,065 patients. Oncotarget 7, 49322-49333 (2016).

\section{Acknowledgements}

Supported by National Key Research and Development Program of China (2016YFC1302700 to C.W.), National Science Fund for Distinguished Young Scholars (81725015 to C.W.), Medical and Health Technology Innovation Project of Chinese Academy of Medical Sciences (2016-I2M-3-019 to D.L.; 2016-I2M-4-002 to C.W.; 2019I2M-2-001 to D.L. and C.W.; 2016-I2M-1-001 to W.T.), Beijing Outstanding Young Scientist Program (BJJWZYJH01201910023027 to C.W.) and National Natural Science Foundation of China (81988101 to D.L. and C.W.).

\section{Author contributions}

C.W., G.G., and D.L. conceptualized and supervised this study. Y. Lin, Y.S., and Y.M. performed bioinformatics analysis. Y. Lin, Y. Luo, and W.G. performed statistical analysis. X.Z. performed in vitro drug vulnerability experiments. Y. Luo and Y.X. prepared DNA and RNA samples. Y.X., M.S., and W.T. responded for clinical data and biospecimen collection and performed experiments. Y. Lin and Y. Luo drafted and C.W., G.G., and D.L. reviewed the manuscript.

\section{Competing interests}

The authors declare no competing interests.

\section{Additional information}

Supplementary information is available for this paper at https://doi.org/10.1038/s41467020-19949-6.

Correspondence and requests for materials should be addressed to G.G. or C.W.

Peer review information Nature Communications thanks Leng Han and the other, anonymous, reviewer(s) for their contribution to the peer review of this work. Peer reviewer reports are available.

Reprints and permission information is available at http://www.nature.com/reprints

Publisher's note Springer Nature remains neutral with regard to jurisdictional claims in published maps and institutional affiliations.

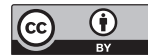

Open Access This article is licensed under a Creative Commons Attribution 4.0 International License, which permits use, sharing, adaptation, distribution and reproduction in any medium or format, as long as you give appropriate credit to the original author(s) and the source, provide a link to the Creative Commons license, and indicate if changes were made. The images or other third party material in this article are included in the article's Creative Commons license, unless indicated otherwise in a credit line to the material. If material is not included in the article's Creative Commons license and your intended use is not permitted by statutory regulation or exceeds the permitted use, you will need to obtain permission directly from the copyright holder. To view a copy of this license, visit http://creativecommons.org/ licenses/by/4.0/.

(c) The Author(s) 2020 